\title{
Sur le passage mathématique de l'Épinomis (990c-992a)
}

Signification et postérité

David Rabouin et Bernard Vitrac

\section{(2) OpenEdition}

\section{Journals}

Édition électronique

URL : https://journals.openedition.org/philosant/2041

DOI : 10.4000/philosant.2041

ISSN : 2648-2789

\section{Éditeur}

Éditions Vrin

\section{Édition imprimée}

Date de publication : 30 octobre 2010

Pagination : 5-39

ISBN : 978-2-7574-0179-8

ISSN : 1634-4561

\section{Référence électronique}

David Rabouin et Bernard Vitrac, «Sur le passage mathématique de l'Épinomis (990c-992a) »,

Philosophie antique [En ligne], 10 | 2010, mis en ligne le 11 juillet 2019, consulté le 02 décembre 2022

URL : http://journals.openedition.org/philosant/2041; DOI : https://doi.org/10.4000/philosant.2041

\section{(c) $(1) \odot$}

Creative Commons - Attribution - Pas d'Utilisation Commerciale - Pas de Modification 4.0 International - CC BY-NC-ND 4.0

https://creativecommons.org/licenses/by-nc-nd/4.0/ 


\section{SUR LE PASSAGE MATHÉMATIQUE DE L'ÉPINOMIS (990C-992 A) : SIGNIFICATION ET POSTÉRITÉ* \\ David RABOUIN \\ CNRS UMR 7219 Laboratoire de philosophie et d'histoire des sciences \\ Bernard VITRAC \\ CNRS UMR 8210 Histoire et anthropologie des mondes antiques}

RÉSUMÉ. Dans cet article, nous analysons le passage dit «mathématique » de l'Épinomis. Dans le programme de formation proposé pour les futurs membres du conseil vespéral de vigilance (990c5-991b4), certains interprètes modernes ont cru voir un témoignage capital pour l'histoire des mathématiques grecques anciennes portant sur la question de l'irrationalité. L'analyse du lexique et du mode de composition du texte - un collage maladroit d'expressions reprises aux loci mathematici platoniciens -, la confrontation avec la littérature mathématique conservée et ce que l'on sait de l'histoire de cette discipline avant Euclide montrent que cette interprétation, pourtant largement répandue, est sans fondement. En revanche, il est possible de reconstituer une intention philosophique compréhensible et mieux à même de rendre compte de certaines particularités $\mathrm{du}$ passage en consultant les commentateurs anciens (Nicomaque de Gérase, Théon de Smyrne, Jamblique de Chalcis, Proclus de Lycie). En privilégiant le passage conclusif sur le «lien» des mathématiques (991d8-992a1), ils identifiaient une tout autre question : celle de l'unité des mathématiques, à partir de laquelle le passage mathématique prenait à leurs yeux tout son sens.

SUMMARY. This paper focuses on the so-called "mathematical passage" of the psendoPlatonic Epinomis. According to some modern scholars, the program proposed for the training of future members of the Nocturnal Council (990c5-991b4) provides crucial testimony of the question surrounding irrationality in ancient Greek mathematics. This commonly held view is shown to be groundless through various means: an analysis of the vocabulary and composition of the text (a clumsy patchwork of expressions taken from

* Une première version de cet article a été présentée le 28 Janvier 2010 dans le cadre du Séminaire «Oralité et Écriture chez Platon» organisé à Montpellier par Jean-Luc Périllié. Nous remercions tou(te)s les participant(e)s pour leurs remarques et critiques. $\mathrm{F}$. Acerbi et $\mathrm{N}$. Vinel ont bien voulu répondre à des questions ponctuelles que nous leur avons posées, en particulier à propos du texte grec du passage que nous étudions ici. Nous remercions également $T$. Bénatouil qui a bien voulu lire une première rédaction de ce texte. 
Platonic loci mathematici); a comparison with the extant mathematical literature and finally a confrontation with the known history of pre-Euclidean mathematics. On the other hand, an examination of ancient commentators (Nicomachus of Gerasa, Theo of Smyrna, Iamblichus of Chalcis, Proclus of Lycia), allows the reconstruction of a possible philosophical intention of the text, thus rendering the passage intelligible. By emphasizing the conclusion of the passage, which deals with the "bond》 of mathematics (991d8-992a1), ancient commentators raised an entirely different question than their modern counterparts: that of the unity of mathematics, which according to the ancients gave the "mathematical passage 》 its overall significance. 
Les mathématiques tiennent dans le développement de l'apocryphe ${ }^{1}$ Épinomis une place de choix. On en trouve dans le dialogue cinq mentions importantes:

1) En 976e-979b3, après l'énumération d'une longue liste de savoirs qui ne sauraient prétendre au titre de sagesse, est mentionnée la science du nombre, condition nécessaire de ladite sagesse et don éminent du plus éminent des Dieux, l'univers lui-même.

2) Reprenant la question de la nature et du nombre des espèces de sagesse recherchée dans le cadre d'un exposé théogonique et zoogonique, l'Athénien, après une très brève allusion (981b3-4) aux cinq polyèdres

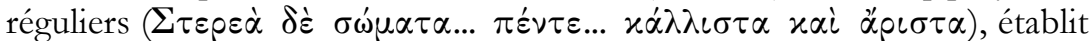
le caractère igné - d'où leur visibilité - et divin des astres (982c5-984a7). Or cette dernière conclusion est inférée grâce aux mathématiques : la régularité de leurs mouvements qu'établit l'astronomie n'est pas mécaniquement explicable. Au contraire, elle justifie qu'on les reconnaisse comme des êtres vivants et intelligents, soit des Dieux en eux-mêmes, soit des $\dot{\alpha} \gamma \alpha \dot{\lambda} \lambda \mu \alpha \tau \alpha$ des Dieux.

3) Des autres espèces de divinités, celles des cultes traditionnels, les démons, les héros ou demi-dieux, associés à l'éther, à l'air et à l'eau, l'Athénien a peu à dire et engage le législateur à respecter la tradition. Car la race humaine est, et restera, ignorante en ce qui concerne des êtres qui sont invisibles, soit en permanence, soit la plupart du temps. Mais c'est tout le contraire avec les huit puissances que sont le soleil, la lune, les Fixes et les cinq planètes. D'où un bref excursus (986a8-988a5) sur les huit révolutions célestes, sur leur lointaine découverte dans les terres d'Égypte et de Syrie au climat propice à l'observation, sur la nécessaire reprise de leur étude et son amélioration par les Grecs.

1. Le dossier complet sur l'authenticité est donné dans la première partie de Tarán 1975. Voir aussi infra n. 46. 
Le terme de ce développement (990a2) permet de répondre (partiellement) à l'une des interrogations du Livre XII des Lois sur ce qui fait l'unité des vertus : la sagesse s'identifie ici à la piété et le nom de cette sagesse est astronomie (990a4)!

4) L'atteindre n'est pas chose facile. Depuis le début du dialogue, on sait que sagesse et bonheur sont réservés à une élite peu nombreuse. Y accéder suppose des natures bien disposées auxquelles il faudra dispenser d'importants enseignements propédeutiques (990c3-5). Ceux-ci sont essentiellement mathématiques : étude du nombre, géométrie, stéréométrie. Ce développement consacré aux disciplines mathématiques (990c5991b5) a suscité de nombreux commentaires, de la part des éditeurs et traducteurs de l'Épinomis bien sûr, des philosophes, mais aussi des historiens des mathématiques, à cause des difficultés textuelles qu'il présente et de l'obscurité de son message. Déjà Paul Tannery, en 1880, sans admettre l'authenticité de l'Épinomis, convoquait ce témoignage pour confirmer la thèse, selon lui platonicienne, que l'unité de la science mathématique réside « dans la construction géométrique de la racine carrée incommensurable (sic)» ou, pour le dire autrement, que ce qui compte dans la géométrie, " c'est l'algèbre $»^{2}$ ! La formulation est totalement anachronique et sans appui textuel chez Platon. Ce genre d'interprétation at-il la moindre pertinence en ce qui concerne notre passage ? C'est ce que nous examinerons en détail dans la deuxième partie de cet article.

5) Les sciences mathématiques reviennent enfin une dernière fois

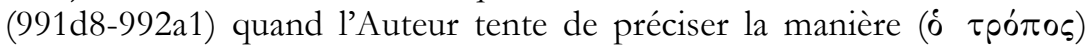
dont ces études doivent être menées, ce qui lui donne l'occasion de souligner l'unité fondamentale de ces sciences.

Dans l'inventaire précédent, le lecteur aura remarqué que «sciences mathématiques » est entendu au sens ancien du terme, donc comme un ensemble de savoirs plus large que celui que retiendrait spontanément un Moderne, incluant des disciplines désormais considérées (et, ce depuis le XVII ${ }^{e}$ siècle au moins) comme relevant de la physique, par exemple l'harmonique ou l'astronomie. C'est même un des traits spécifiques des mathématiques grecques anciennes par contraste avec celles qu'ont développées d'autres civilisations antiques. Elles sont irréductiblement plurielles et constituées en disciplines plus ou moins autonomes: arithmétique, harmonique, géométrie, astronomie, optique, mécanique... Cela vaut surtout pour les descriptions philosophiques impliquant une «classification» des sciences, mais c'est aussi vrai si l'on prend comme

2. Tannery 1925 ; la seconde citation se trouve p. 17 , la première, p. 20. Cette dernière est reproduite sans le moindre esprit critique par des Places 1935, p. 550. 
critère la répartition des écrits conservés ${ }^{3}$. Les mathématiques avaient donc, en Grèce ancienne, un usage instrumental qui débordait les besoins pratiques (comptabilité, arpentage, architecture), usage fondamental pour la compréhension de certains phénomènes physiques. Rien de comparable - exception faite de l'astronomie - n'a existé dans les autres civilisations anciennes. Malgré la fréquente identité des acteurs dans ces différentes disciplines - Euclide, par exemple, a consacré ses travaux à l'arithmétique, la géométrie, l'optique, la catoptrique, l'harmonique, l'astronomie -, cet "encyclopédisme » mathématique faisait que la pluralité des mathématiques était une donnée culturelle. Leur « unité » était donc un problème pour le philosophe qui voulait y réfléchir.

L'ultime mention (5), précisément parce qu'elle permettait de dessiner les contours d'un programme d'unité des mathématiques que les lecteurs de Platon étaient bien en mal de discerner dans son œuvre - malgré les appels de leur maître à la saisie de ce qui fait la «communauté » et la " parenté » des mathématiques -, a été mobilisée à plusieurs reprises dans l'Antiquité. Ce sera l'objet de notre troisième partie que d'étudier ces lectures et de faire revivre une interprétation proprement philosophique du passage mathématique de l'Épinomis. Mais avant d'y entrer de plainpied, il convient de faire quelques remarques sur le contexte dans lequel se situent nos deux passages.

\section{L'héritage platonicien de l'Épinomis}

L'Auteur de l'Épinomis n'a pas manqué de s'inspirer de loci mathématiques authentiquement platoniciens ${ }^{4}$. C'est tout à fait évident dans les mentions (1) et (2) :

- Les valeurs du nombre se déduisent des défauts qu'impliquerait son absence : il manifeste l'ordre, donne une forme, un rythme, une harmonie. Plus généralement, il est un élément fondamental de compréhension qui permet de "rendre compte » ( est donc bien une condition nécessaire de la sagesse (cf. Lois, VII, 817bc). C'est même un critère de distinction avec l'animal; supprimer le nombre réduirait l'homme à l'état de bête. Cela entraînerait la disparition de tous les arts (cf. République, VII, 522c1-3, e5 ; Pbilèbe, 55e).

3. Voir Vitrac 2005.

4. Nous nous contentons ici de quelques rappels sommaires concernant seulement les mathématiques. Le lecteur intéressé trouvera une comparaison détaillée des doctrines exposées dans l'Épinomis avec Platon, de leurs traits communs et de leurs écarts, dans l'abondante introduction et les notes de commentaire de Tarán 1975. 
- Le nombre est un don divin. Le mythe le disait (Eschyle, Prométhée enchaîné, v. 459 ; Platon, Phèdre, 274c8 ; Philèbe, 16c4-6); mais surtout la cosmogenèse du Timée a expliqué comment la mise en place des orbes célestes par le Démiurge visait, entre autres, à permettre aux hommes de concevoir le nombre et d'apprendre à compter (39b2-c5).

- Que la régularité des mouvements célestes ne puisse s'expliquer dans le cadre de théories mécanistes, mais implique l'intelligence des astres et la présence d'une finalité cosmique se trouve également dans le Timée et constitue l'argument principal dans le réquisitoire du Livre X des Lois contre les cosmologies matérialistes (896e8-899d3).

- L'appariement entre les constituants fondamentaux (Feu, Air, Eau, Terre) et quatre des cinq figures solides régulières (pyramide, octaèdre, icosaèdre, cube) est un autre des traits saillants de la cosmogenèse du Timée (53c5-57d6). L'idée est ici reprise et, semble-t-il, systématisée puisqu'un cinquième constituant, l'Éther, est introduit, sans doute associé au dodécaèdre que Platon attribuait à la figure globale du cosmos ; cela dit, l'Auteur de l'Épinomis ne dit rien de précis à ce sujet. Il ne fournit aucune description géométrique - même sommaire - dudit polyèdre, contrairement à ce qu'avait fait Platon en termes de faces, d'angles solides et de triangles constitutifs. C'est un peu étonnant de la part de quelqu'un qui tient les mathématiques en si haute estime. Peut-être n'a-til pas voulu insister sur l'hétérodoxie de cette thèse par rapport au Timée. Peut-être ses (in)compétences en géométrie ne lui permettaient-elles pas d'en dire davantage.

Un écart autrement important avec le platonisme que nous connaissons par les dialogues est évidemment la position de l'astronomie comme sagesse et point d'aboutissement de la formation des futurs

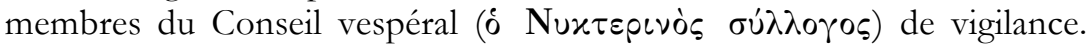
Dans la République, l'astronomie n'était qu'une des sciences mathématiques propédeutiques. Il n'est même pas certain qu'elle était la plus importante : ce dialogue mettait plutôt l'accent sur l'importance philosophique de l'arithmétique (qui permet de saisir les problèmes relatifs à l'Un et au Multiple) et Socrate enjoignait aux astronomes de s'inspirer des géomètres quant à leur méthode d'investigation (530b7-c3). Le couronnement de la formation des futurs gardiens philosophes de la Cité n'était pas de type mathématique, mais résidait dans la pratique de la dialectique, seule démarche anhypothétique. Dans le "programme » de l'Épinomis, la dialectique n'a pas conservé cette éminente fonction. Elle est à peine évoquée à la fin du dialogue, comme une sorte de méthode d'investigation quelque peu scolaire (991c2-6). 


\section{Les études propédeutiques : 990c5-991b4}

Commençons par proposer une traduction de ce passage, découpé en segments numérotés pour faciliter la discussion qui suit :

\begin{tabular}{|c|c|c|}
\hline (1) & 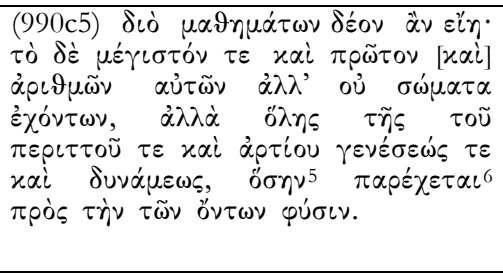 & $\begin{array}{l}\text { C'est pourquoi il faut qu'il y ait des } \\
\text { études mathématiques; la plus im- } \\
\text { portante et la première sera celle des } \\
\text { nombres eux-mêmes, non pas ceux qui } \\
\text { ont un corps, mais de toute la géné- } \\
\text { ration et la puissance de l'impair et du } \\
\text { pair, pour autant que cela s'applique à } \\
\text { la nature des êtres. }\end{array}$ \\
\hline (2) & 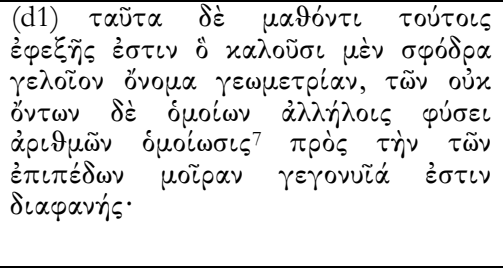 & $\begin{array}{l}\text { Et pour celui qui a étudié ces choses } \\
\text { vient à leur suite celle qu'on appelle du } \\
\text { nom tout à fait ridicule de "géo- } \\
\text { métrie », quoique une homoiosis des } \\
\text { nombres qui, par nature, ne sont pas } \\
\text { semblables les uns aux autres - établie } \\
\text { par comparaison avec ce qui est propre } \\
\text { aux surfaces -, soit transparente; }\end{array}$ \\
\hline (3) & 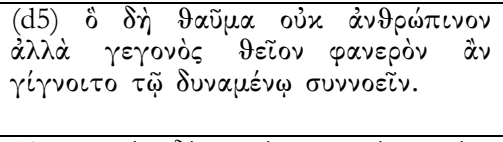 & $\begin{array}{l}\text { pour celui qui est capable de le con- } \\
\text { cevoir, il sera alors manifeste qu'une } \\
\text { telle merveille ne saurait être humaine, } \\
\text { mais que sa réalisation est divine. }\end{array}$ \\
\hline (4) & 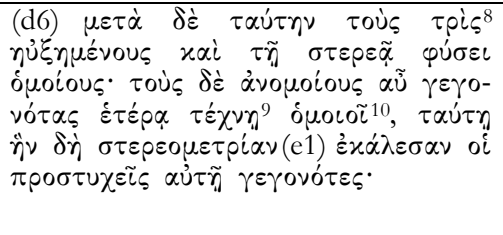 & $\begin{array}{l}\text { Après celle-ci [il devra étudier] les nom- } \\
\text { bres ayant été triplement accrus, sem- } \\
\text { blables quant à leur nature solide. Mais, } \\
\text { pour ceux qui se trouvent dissem- } \\
\text { blables, il y a encore assimilation par un } \\
\text { autre art, celui que ceux qui en ont fait } \\
\text { l'expérience ont appelé «stéréométrie ». }\end{array}$ \\
\hline
\end{tabular}

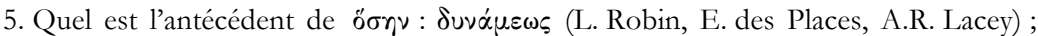

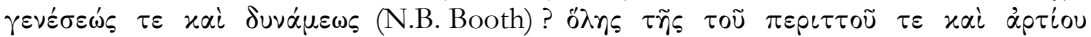

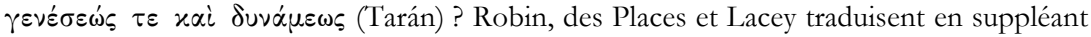

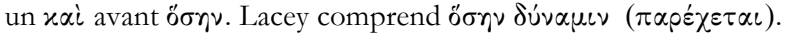

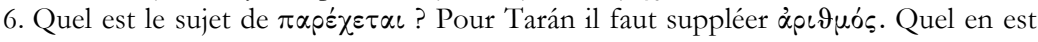
le sens : avoir tel effet, avoir de l'influence, avoir de la puissance sur, communiquer...

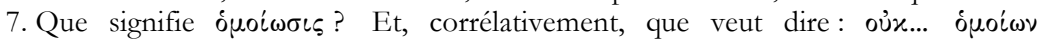

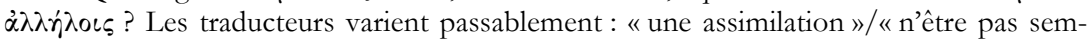
blables » (Robin, Tarán) ; «la possibilité de la comparaison»/ "n'être pas comparables » (des Places). Voir aussi infra n. 36. Le sens est-il le même quatre lignes plus bas: ( $\tau \tilde{n}$

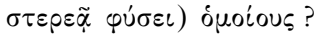

8. $\tau$ pis Bekker, $\tau \rho \tilde{i} \varsigma$ AO.

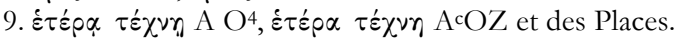

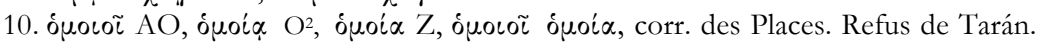

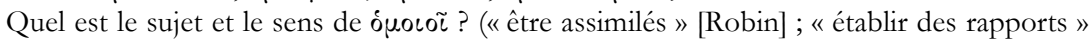
[des Places]). 


\begin{tabular}{|c|c|c|}
\hline (5) & 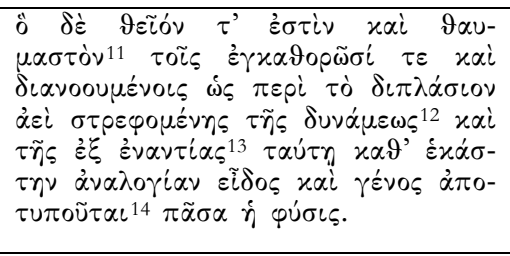 & $\begin{array}{l}\text { Mais ce qui est divin et merveilleux, } \\
\text { pour ceux qui le remarquent et y } \\
\text { réfléchissent, c'est de voir comment, la } \\
\text { puissance et son opposée tournant et se } \\
\text { retournant toujours autour du double, } \\
\text { la nature tout entière se façonne en } \\
\text { espèce et genre, selon chaque médiété : }\end{array}$ \\
\hline (6) & 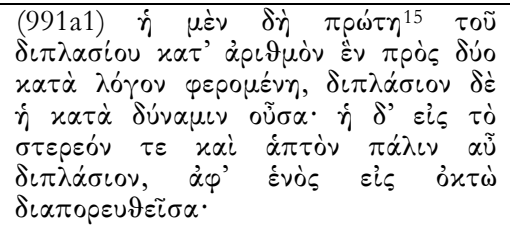 & $\begin{array}{l}\text { - d'une part, la première, du double } \\
\text { selon le nombre, menant un vers deux } \\
\text { selon le rapport, puis, double, étant } \\
\text { selon la puissance, puis, à nouveau et à } \\
\text { son tour double en direction de ce qui } \\
\text { est solide et tangible, étant passée de un } \\
\text { jusqu'à huit. }\end{array}$ \\
\hline (7) & 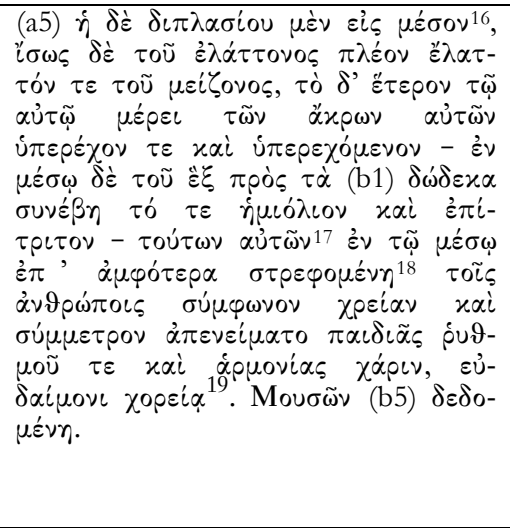 & $\begin{array}{l}\text { - d'autre part, vers un médian du } \\
\text { double, l'une, excédant le plus petit [des } \\
\text { termes] et étant plus petite que le plus } \\
\text { grand d'une manière égale, l'autre } \\
\text { dépassant et étant dépassée par la } \\
\text { même partie de ses extrêmes - et, en } \\
\text { tant que moyen de six à douze, se } \\
\text { trouvent et l'hémiole et l'épitrite -, } \\
\text { tournant et se retournant de part et } \\
\text { d'autre, au milieu de ceux-là mêmes, } \\
\text { elle a donné en partage aux hommes un } \\
\text { usage consonant et proportionné en } \\
\text { vue d'un jeu de rythme et d'harmonie, } \\
\text { offrande au chour bienheureux des } \\
\text { Muses. }\end{array}$ \\
\hline
\end{tabular}

11. La version théonienne (10.7-11 Hiller) de 990d6-e2 est assez divergente.

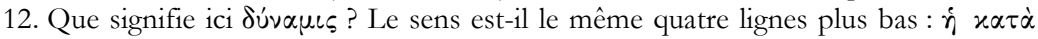

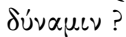

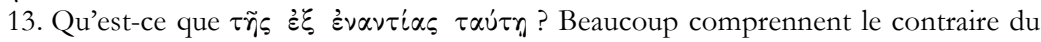
double, donc la moitié ; mais c'est confondre « contraire » et « inverse ».

14. Quel est le sujet de $\dot{\alpha} \pi \circ \tau \cup \pi \circ \tilde{u} \tau \alpha \iota$ ? Pour Robin, des Places et Tarán, c'est $\pi \tilde{\alpha} \sigma \alpha$

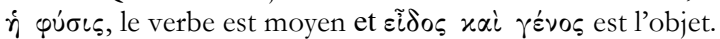

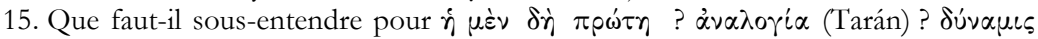
(Lacey) ? Même chose pour les deux occurrences à la ligne suivante. Faut-il comprendre

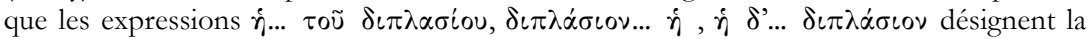

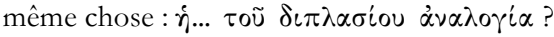

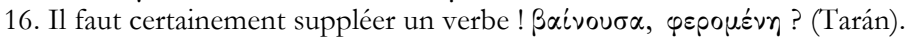

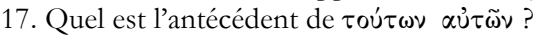

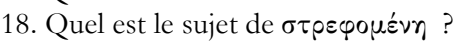

19. Ambiguiité du datif : donné au chœur (Robin, Lacey), donné par le chœur (des Places, Tarán). 
Notre traduction ne vise ni l'élégance littéraire, ni l'explicitation de ce que le grec maintient dans l'ambiguité. S'il consulte les notes infrapaginales que nous avons insérées, le lecteur constatera aisément que le passage est souvent grammaticalement imprécis, en particulier dans sa syntaxe, qu'il multiplie les sous-entendus, voire emploie les mêmes termes, y compris lorsqu'ils possèdent une acception technique ( $\alpha^{2} v \alpha \lambda o \gamma i \alpha, \delta \dot{-}$ vauıs, ónoio ), dans des sens différents. Ce lexique n'est pas toujours très clair et nous discuterons un certain nombre d'exemples qui touchent

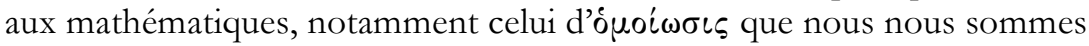
contentés de translittérer.

La plupart des interprètes admettent que le texte grec n'est probablement pas bien transmis et que le style en est négligé. C'est possible, mais à l'inverse on peut observer que l'emphase stylistique n'est pas exclue. Voyez, par exemple, les cinq occurrences du verbe $\gamma^{\prime} \gamma^{\prime}$ voua $(\gamma \varepsilon$ -

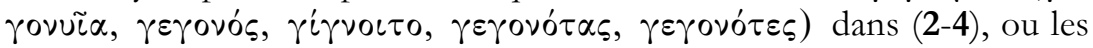
cinq mentions de $\tau \dot{o} \delta \iota \pi \lambda \alpha \dot{\sigma} \iota \mathbf{v}$ dans (5-7). L'obscurité du massage n'est pas non plus sans rappeler celle du célèbre couplet sur le nombre nuptial dans la République ${ }^{20}$.

\section{Le programme de formation}

Un autre rapprochement possible avec le même dialogue, mais cette fois avec le Livre VII, tient à ce que notre passage se présente - au moins dans les items (1-2-4) - comme un programme d'études. La triade arithmétique-géométrie-stéréométrie est commune et on pourrait poursuivre le parallélisme en rapportant la section (5-7) à l'harmonique. On retrouverait ainsi les sciences du quadrivium, étant entendu, comme nous l'avons déjà dit, que l'astronomie n'est plus ici considérée comme une discipline propédeutique à la dialectique, mais comme le couronnement des études scientifiques. Luc Brisson a suggéré que cette correspondance entre programmes de formation a pu jouer un rôle dans l'attribution de l'un des sous-titres de l'Épinomis rapportés à Thrasylle par Diogène Laërce : le Philosophe ${ }^{21}$. Il est bien vrai que, par contraste avec le curriculum proposé au Livre VII des Lois, cette fois pour l'ensemble des hommes libres de la

20. Resp. VIII, 546b4-c7. En termes mathématiques modernes, il s'agit de décrire des triplets dits pythagoriciens (triplets de nombres entiers $(a, b, c)$ vérifiant $\mathrm{a}^{2}=\mathrm{b}^{2}+\mathrm{c}^{2}$ ) et d'observer que si l'on part d'un premier triplet [dans le cas de Platon $(3,4,5)$ ], que l'on forme les nombres solides $M=a^{3}, N=a^{2} b, O=a b^{2}, P=b^{3}$, nombres en progression continue dans le rapport de a à b, puis les nombres solides $\mathrm{A}=\mathrm{M}+\mathrm{O}, \mathrm{B}=\mathrm{N}+\mathrm{P}, \mathrm{C}$ $=\mathrm{c}^{3}$ [respectivement 27, 36, 48, 64, rapport épitrite (4/3), puis $(75,100,125)$ dans l'exemple de Platon], le triplet (A, B, C) est aussi pythagoricien et «semblable » au premier. La terminologie du passage, en termes de "triangles rectangles en nombres ", est mixte, à la fois arithmétique et géométrique.

21. Brisson 2005, p. 15-16. 


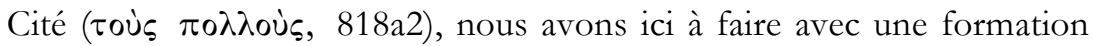
théorique:

- l'étude du pair et de l'impair portera sur les «nombres eux-mêmes,

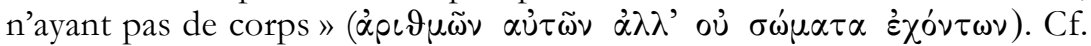

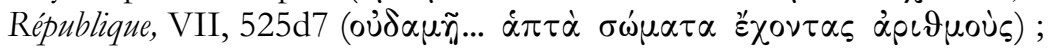

- l'Auteur souligne que le nom de la «géo-métrie » est ridicule (ö $x \alpha$ -

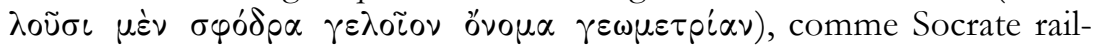
lait le mode d'expression "figurée» de cette discipline (cf. République,

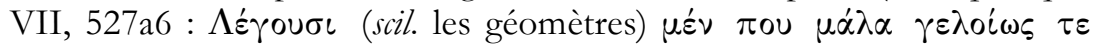
x $\left.i_{i} \alpha \nu \alpha \gamma \varkappa \alpha i \omega \varsigma\right)$, tandis que les Lois, se gardant d'introduire aucune désignation savante des sciences, soulignaient au contraire la dimension métrologique du deuxième des trois objets d'étude 22.

- Quant à la section (5-7) consacrée à l'harmonique - si c'est bien de cela qu'il s'agit -, il va de soi qu'elle est théorique ; comme le Timée qui l'a inspirée, elle a même une portée cosmique.

Observons toutefois qu'à la différence du programme authentiquement platonicien du Livre VII de la République, la description des disciplines mathématiques par l'Épinomis relève plutôt de l'«anticlassification » en ce sens qu'elle vise à gommer les oppositions qui gouvernent le quadrivium («science de ce qui est en repos/science de ce qui est en mouvement »; " discret/continu »; « visible/audible ») plutôt qu'à les souligner. L'Auteur essaie d'établir une continuité entre arithmétique et géométrie ; la description de l'harmonie se réduit à la théorie des médiétés entre nombres (en reprenant un schéma employé dans le Timée pour décrire la construction de l'Âme du Monde) et l'astronomie culmine avec la connaissance des périodes des révolutions célestes, autrement dit, là aussi, avec la connaissance d'un système de nombres. Dès lors, l'astronomie n'est que le sommet d'une unique pyramide qui représenterait une science mathématique fondamentale et quasi unique : la science des nombres.

\section{Le lexique géométrique}

Dans un tel cadre fondamentalement arithmétisant, il était difficile, sans être très réducteur, de faire entrer la géométrie. De fait, c'est bien ce qui se produit dans les assertions (2) et (4) : la caractérisation de la géométrie est extrêmement sommaire. Qui pis est, la signification exacte de cette portion n'est pas claire. Analysons pour commencer le lexique mathématique ou paramathématique de notre passage : il contient en effet un certain nombre de termes qui figurent dans la plus ancienne littérature mathématique grecque conservée, notamment les Éléments d'Euclide. 


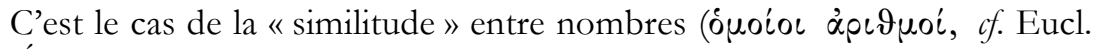
Él. Df. VII 22) : l'expression apparait ici en $990 \mathrm{~d} 2$ (en d7-8, on trouve la

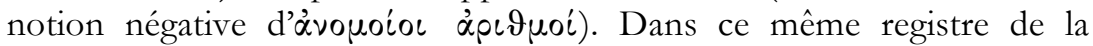

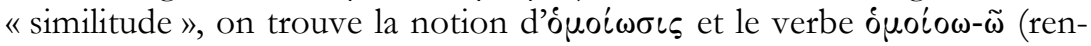
dre semblables ?), termes que les mathématiciens n'utilisent pas, mais qui sont ici sans doute employés dans une acception technique. D'ailleurs Platon lui-même, dans le passage déjà mentionné sur le nombre nuptial,

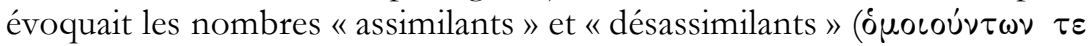

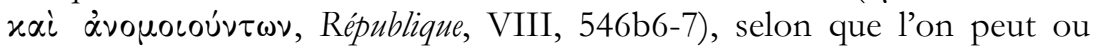
non les décomposer en produits de trois facteurs égaux.

À côté de cette thématique de la similitude, l'autre notion mathématique mise en œuvre ici est tout simplement la distinction «plan(e)/soli-

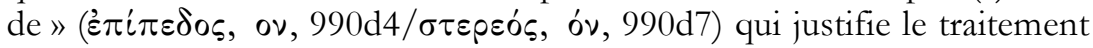
successif de deux (branches de la même) discipline : géométrie (2) et stéréométrie (4), opposition dont il faut souligner :

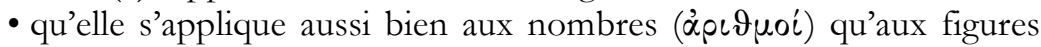
$\left(\sigma \chi \eta_{\eta}^{\prime} \mu \alpha \tau \alpha\right)$, car elle est associée à une représentation géométrique multiplicative ${ }^{23}$ des nombres (en principe non premiers), produits de deux ou trois facteurs ( $c f$. Eucl. Él. Df. VII, 17-18) que l’on peut considérer métaphoriquement comme des surfaces ou des volumes ;

- que cette modalité de représentation - et la dualité ṡ $\pi i$ $\pi \varepsilon \delta \circ \varsigma / \sigma \tau \varepsilon \rho \varepsilon \delta_{\varsigma}$ - est bien connue de Platon; on les trouve dans le programme de République VII ${ }^{24}$, dans le passage mathématique du Théétète

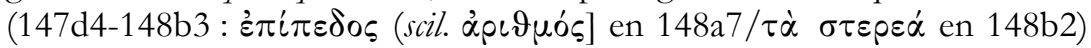
et elle gouverne l'assertion problématique de Timée 32a7-b3 qui a fait couler tant d'encre ${ }^{25}$;

23. Pour la distinguer de la représentation additive des nombres en tant que nombres dits polygones que Nicomaque et Théon de Smyrne présentent comme une doctrine propre à l'ancien pythagorisme et dont on trouve en effet des échos à l'époque classique (Aristote, Speusippe).

24. Le nom «stéréométrie » n’apparaît pas dans le Livre VII de la République, mais la distinction géométrie plane/solide est tout à fait explicite; on peut même dire qu'elle est mise en scène par Socrate quand il feint d'avoir oublié cette distinction $(M \varepsilon \tau \dot{\alpha} \varepsilon \dot{\varepsilon} \pi i \pi \varepsilon \delta o v$,

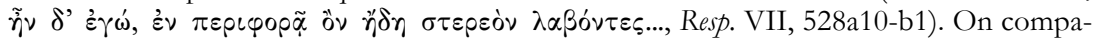

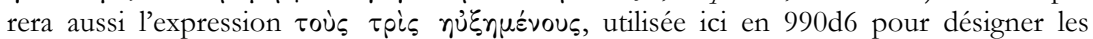

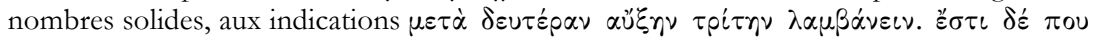

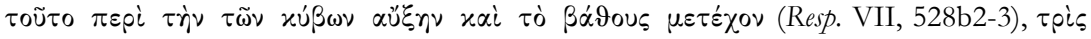

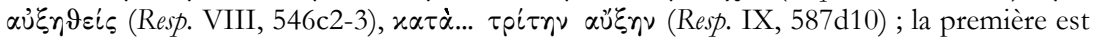
géométrique, les deux autres portent sur les nombres.

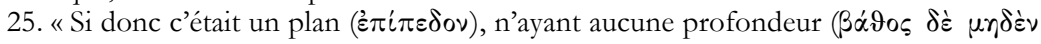

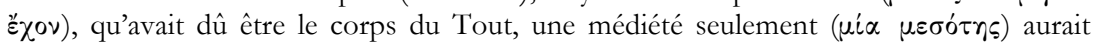
suffit à lier les termes qui l'accompagnent ainsi qu'elle-même; maintenant, il était convenu qu'il devait être de forme solide ( $\sigma \tau \varepsilon \rho \varepsilon 0 \varepsilon \iota \delta \tilde{\eta})$, et les solides, ce n'est jamais une seule, mais toujours deux médiétés qui les harmonisent $\left(\begin{array}{clll}\tau \dot{\alpha} & \delta \dot{\varepsilon} & \sigma \tau \varepsilon \rho \varepsilon \dot{\alpha} & \mu i \alpha\end{array} \mu \dot{\varepsilon} \nu\right.$ 
- qu'il s'agit d'une "métaphore » ou d'une analogie (!) - aussi bien chez Euclide que dans le passage du Théétète lequel, pour la signifier, utilise le verbe $\dot{\alpha} \pi \varepsilon \iota x \dot{\alpha} \zeta_{\omega}$ (représenter) -, mais d'une analogie métrologiquement fonctionnelle.

\section{Similitude et irrationalité}

Avant de revenir à l'interprétation du passage de l'Épinomis, ouvrons une parenthèse afin que le lecteur possède tous les éléments de la discussion : un nombre plan (resp. solide) peut être conçu comme la mesure d'une aire rectangulaire (resp. d'un solide parallélépipédique rectangle) car la surface (resp. le volume) d'une telle aire (resp. d'un tel solide) est le produit des mesures de la longueur et de la largeur (resp. de la longueur, de la largeur et de la profondeur), mesurées par une certaine unité. Bien entendu, si les facteurs du nombre plan sont égaux entre eux ( $\tau \dot{\nu} \nu \mu \dot{\varepsilon} \nu$

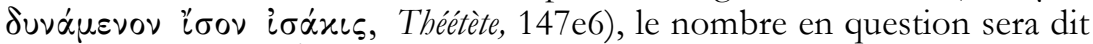
"carré » ( $f$. Eucl. Él. Df. VII, 19) et la figure géométrique associée sera

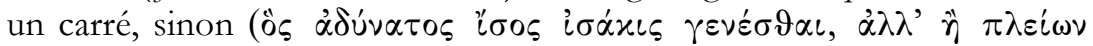

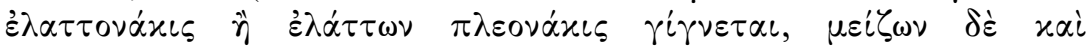

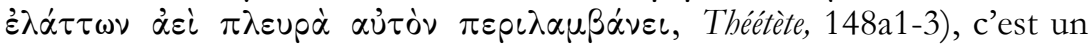
nombre promèque et la figure associée est un rectangle. L'approche de Théétète, comme celle du Livre X des Éléments d'Euclide, coordonne donc arithmétique et géométrie à l'aide d'un langage «commun» ("plan », « solide », "carré », " cube », " côté »...), malgré l'irréductible incommunicabilité des genres auxquels les objets de ces deux sciences appartiennent : nombres (discret)/grandeurs (continu).

Mais quelques complications surgissent :

- à l'exception des nombres premiers, la composition multiplicative d'un nombre n'est pas unique : 36 peut être considéré comme un nombre carré $(6 \times 6)$ ou rectangle $(4 \times 9)$. Dans la représentation, il est donc possible de lui associer un carré ou un rectangle. C'est pourquoi Théétète

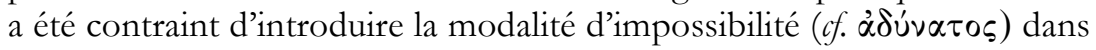
ses définitions. La correspondance ainsi posée entre arithmétique et géométrie n'est pas biunivoque : tout nombre entier peut être représenté comme la mesure d'une entité géométrique (ligne, surface, solide) et ce, de plusieurs façons ${ }^{26}$;

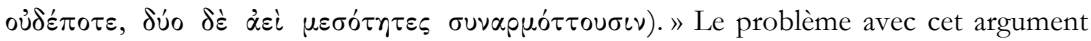
"de convenance » est que la dernière assertion, à strictement parler, est fausse. Voir Vitrac 2006, en particulier p. 39-58.

26. Si on comprend la distinction introduite par Théétète pour les nombres non carrés comme une implication, il faudra accepter qu'un nombre premier p puisse être considéré comme $1 \times \mathrm{p}$ (nombre plan) et $1 \times 1 \times \mathrm{p}$ (nombre solide) avec l'unité pour l'un (au moins) de ses côtés. Les Df. euclidiennes, en principe, excluent que l'unité soit un nom- 
- pire encore, il existe des aires simples, même rectangulaires, qui ne correspondent à aucun entier (par exemple le rectangle contenu par la diagonale d'un carré unitaire comme longueur et son côté comme largeur $^{27}$ );

- même pour les aires rectangulaires qui admettent une expression numérique (donc entière, grâce à un choix judicieux d'unité), la question se posera de savoir s'il peut ou non s'agir d'un nombre carré. En effet, géométriquement (à la règle et au compas), il est très facile de «transformer » n'importe quel rectangle en un carré (Théétète et Euclide

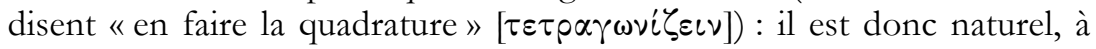
cause de la pluralité des décompositions possibles, de se demander si, à cette nouvelle aire carrée, équivalant au rectangle initial, correspond un nombre irréductiblement promèque ou s'il peut s'agir d'un nombre carré. Si c'est impossible, le côté du carré équivalent est donc incommensurable

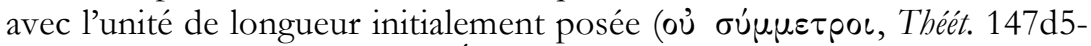

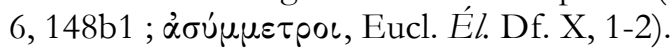

Comme l'a bien compris Théétète, la correspondance arithmétique/géométrie, même si elle n'est pas biunivoque, permet déjà de distinguer d'emblée (au moins) deux catégories de segments de droites

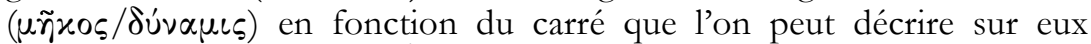
(Théetète, 148b2 ; cf. Eucl. Él. Df. X, 4). Que l'orientation des travaux de Théétète sur les (lignes) irrationnelles ait été classificatoire, nous en avons la confirmation, indépendamment du dialogue de Platon, par le témoignage de Pappus s'appuyant sur l'historien des mathématiques péripatéticien Eudème de Rhodes ${ }^{28}$.

\section{L'absence de l'irrationalité dans l'Épinomis}

Fermons cette parenthèse sur la classification des irrationnelles, et revenons à la signification de notre passage de l'Épinomis. A-t-elle à voir avec l'irrationalité comme le suggérait déjà Tannery que nous citions en commençant et bien d'autres, qui ont été plus loin encore, notamment A.E. Taylor quand il écrit :

The text of 990 5-991b 4, the most important mathematical passage in the Platonic corpus, is unfortunately uncertain, in part probably corrupted, in part also

bre (i.e. une pluralité d'unités), donc que les nombres premiers soient dits plans ou solides.

27. En termes modernes, son aire est $\sqrt{2}$. $C^{2}$, en notant $C$ la mesure du côté du carré initial, et c'est un nombre irrationnel. Si on transforme géométriquement ce rectangle en carré, le côté de ce dernier (en termes modernes: $\sqrt[4]{2}$.C), correspond aux lignes irrationnelles qu'Euclide appelle « médiales ».

28. Pappus-Thomson 1930, Livre I, \1, p. $63=$ Wehrli 1969, fragments $N^{\circ} 141$ I, II. 
possibly never reduced to grammatical form by the writer, but the sense is clear. The point of chief significance is the revolutionary demand that quadratic and cubic "surds" shall be recognized as numbers in opposition to the traditional view that there are "irrationnal" magnitudes (lengths, areas, volumes), but no "irrational" number29.

La revendication concernant les nombres irrationnels que notre texte est censé exprimer serait en effet révolutionnaire : aucun mathématicien grec ancien connu de nous n'y a adhéré. Tous sont restés fidèles à la vision « traditionnelle » (entendez celle que l'on voit mise en œuvre dans le Théetète, développée par Euclide, puis par Apollonius) qui fait de l'incommensurabilité et de l'irrationalité une caractéristique propre aux grandeurs géométriques.

Notre examen du lexique fait fortement douter d'une lecture aussi enthousiaste. Le lexique platonicien de l'irrationalité est déjà assez riche car les dialogues mentionnent la question à sept reprises au moins ${ }^{30}$ et, comme nous l'avons signalé en passant, ledit lexique recoupe (partiellement) celui des Éléments d'Euclide ${ }^{31}$. C'est d'ailleurs l'une des raisons pour lesquelles les historiens des mathématiques accordent un tel intérêt aux loci mathematici des dialogues et, de fait, cette comparaison «PlatonEuclide » remonte à l'Antiquité32. Or aucun des termes de ce lexique ne

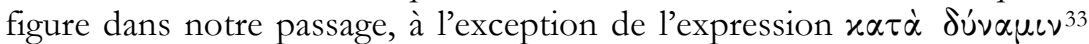
qui figure dans (6), mais ni dans (2), ni dans (4)! Expression dont le champ d'utilisation déborde au demeurant la question des irrationnelles (dans Rép. IX, 587d10 il s'agit clairement d'envisager des nombres élevés à la deuxième puissance) $)^{34}$. Au bout du compte, la «présence» de l'irrationalité dans notre passage n'est qu'un artefact exégétique ou, du moins, le résultat d'inférences incertaines fondées sur la compréhension

29. Taylor 1926, p. 501 n. 1.

30. Hipp. mai. 303b4-c2 ; Resp. 534d5; Resp. 546b4-c8; Theaet. 147d4-148 b3 ; Polit. 266a-b ; Tim. 54b2-5 ; Leg. 819d1-820d6 ; à ces sept loci, on peut adjoindre Resp. 587d411, pour la terminologie. En revanche, comme l'a montré Vinel 2003, on peut écarter Tim. 31c1-32c2 : l'occurrence de Súvauss en 31c5 n'a rien à voir avec l'irrationalité.

31. Outre les termes mentionnés à propos du Théetète, ce lexique commun inclut

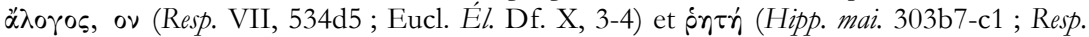
VIII, 546c1, c4; Eucl. loc. cit.). En revanche àpprín (qu'utilise Platon) ne se trouve pas dans les Éléments et le sens de Súvaurs dans le passage du Théetète n'est pas le même que

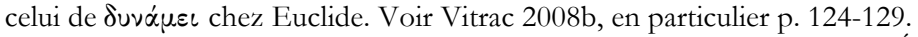

32. Commentaire anonyme du Théétète, commentaire du Livre $\mathrm{X}$ des Éléments attribué à Pappus, ainsi que différentes scholies portées dans les marges des manuscrits d'Euclide.

33. Cf. Epin. 991a3 et Resp. IX, 587d10 ; Polit. 266b5-6 ; Tim. 54b5. Sur ce vocabulaire, voir Vitrac 2008b.

34. Voir Vitrac 2008b, en particulier p. 121-123. 


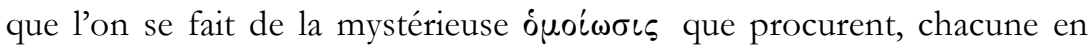
leur domaine, géométrie et stéréométrie.

Nous avons déjà fait le rapprochement entre l'occurrence du verbe

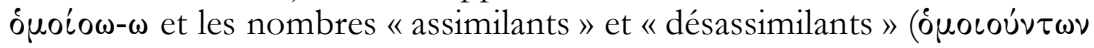

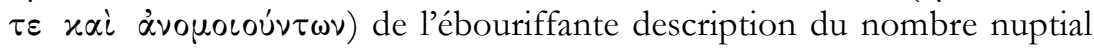
(Rép. VIII, 546b6-7), nombres que l'on peut décomposer en produits de trois facteurs égaux ou non. Ainsi le nombre 48 peut être décomposé de plusieurs manières en tant que nombre solide : 1 x 1 x 48, 1 x 2 x $24 \ldots 2$ x $2 \times 12,2 \times 3 \times 8 \ldots$ Parmi toutes ces décompositions, $3 \times 4 \times 4$ est la plus assimilante car, interprétée géométriquement, elle correspond au parallélépipède rectangle le plus proche du cube que l'on puisse obtenir avec les facteurs de 48. À ce moment de la description du texte de République VIII, il n'est aucunement question d'irrationalité ${ }^{35}$, ni réellement de géométrie, mais des possibilités de décomposition des nombres solides en produits de trois facteurs.

Comme dans le passage du Théétète pour les nombres plans (« quarrables» ou non), il s'agit donc d'une notion de «similarité interne ». Même si les deux notions renvoient à une représentation géométrique multiplicative des nombres, cette similarité des facteurs ne coïncide pas avec la relation de similitude euclidienne qui est une relation binaire entre nombres. Elle en constitue tout au plus un cas particulier : la similitude à un nombre carré pour les nombres plans, à un nombre cube pour les nombres solides. La notion « euclidienne» de «nombres semblables » (Df. VII, 22) n'est peut-être pas une invention d'Euclide - de fait nous n'en connaissons pas l'origine - mais, à notre connaissance, elle ne se trouve ni dans les dialogues de Platon, ni chez Aristote. Bon nombre d'interprètes (Tannery, Jones, Van der Waerden, Lacey, Tarán), ont cru néanmoins la reconnaître dans les nombres semblables ou dissemblables (ónoíous/ávouoíous) de l'Épinomis (990d3, d7) et, à partir de là, ont lu le passage dans l'optique de l'irrationalité ${ }^{36}$. De fait, chez Euclide, la notion de nombres semblables sert à caractériser une classe de

35. Celle-ci intervient seulement après (en 546c4-6), dans une double désignation volontairement énigmatique (« cent des nombres sur les carrés des diagonales exprimables

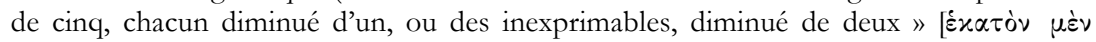

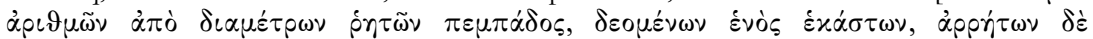
$\delta$ บõ̌v]) du nombre 4800 comme $4800=100 .(49-1)$ et $4800=100 .(50-2)$.

36. À cet égard des Places 1935, p. 548-549 est incohérent et incapable de trancher : il mentionne le sens euclidien de ópoío , citant Tannery et Jones, mais aussi Raeder, qui préconisait de traduire par commensurables! Dans son édition (Des Places 1956), il traduit «comparables» et explique dans la notice (p. 122) que «le mot öuocos et ses composés ou dérivés... ne peuvent exclure ni la notion de proportion, ni celle de commensurabilité ». La confusion est totale! 
rapports numériques qui, elle-même, joue un rôle fondamental dans la classification des lignes irrationnelles. Le Livre X privilégie en effet les aires qui sont l'une relativement à l'autre dans un rapport numérique particulier : celui de nombre carré à nombre carré. Petite subtilité : si deux nombres sont des carrés, leur rapport est évidemment celui d'un carré à un carré, mais l'inverse n'est pas vrai ; ainsi $(50,18)$ ne sont pas des carrés, mais leur rapport est le même que celui de 25 à 9 : il s'agit donc d'un rapport de nombre carré à nombre carré. Il est alors naturel de vouloir caractériser les couples de nombres dont le rapport est celui d'un nombre carré à un nombre carré ; et il se trouve que c'est le cas si et seulement si ces nombres sont plans semblables. Euclide démontre aussi que deux nombres plans sont semblables si et seulement si un nombre

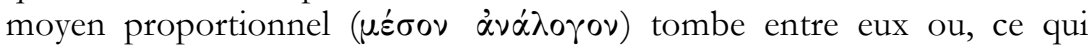
revient au même, si et seulement si leur produit est un carré. Le lecteur vérifiera aisément qu'entre 50 et 18 tombe le moyen proportionnel 30 , et que leur produit 50 x $18=900$ est bien un carré (celui de 30 évidemment ${ }^{37}$.

Les inférences des Modernes sont donc: (i) que les nombres semblables mentionnés en $990 \mathrm{~d} 2$ correspondent à la notion euclidienne; (ii) que, puisque les nombres semblables sont utilisés par Euclide pour caractériser la notion de rapport de nombre carré à nombre carré, ellemême mise en œuvre dans sa classification des irrationnelles, l'Auteur de l'Épinomis, dans (2) et (4), parle - allusivement ! - d'irrationalité. Inférences incertaines et certainement inutiles :

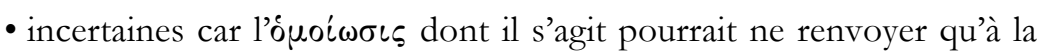
similarité «interne » d'un nombre plan ou solide, mise en œuvre dans les loci du Théetète et de République VIII. Jamblique observe que "les Anciens nommaient ceux-ci (scil. les nombres carrés) "identiques" et "semblables" (ónoíous), à cause de la similitude et de l'égalité de leurs côtés, et, pour les raisons opposées, nommaient les hétéromèques "dissemblables" (àvouoíous) et "autres" »38. En outre, le terme devrait désigner un processus. Or la similitude euclidienne (notion arithmétique) n'est pas un processus, mais une relation déterminée : deux nombres donnés sont semblables ou ne le sont pas. Il n'y a aucune échappatoire à cela, aucune manière de « rendre semblables » au sens euclidien des nombres qui ne le sont pas. On peut certes leur associer des aires rectangulaires, en changer

37. La notion de nombres solides semblables se manipule un peu moins bien, mais Euclide établit tout de même que deux nombres solides sont semblables si et seulement si deux nombres moyens proportionnels tombent entre eux (Él. VIII 19, 21) et que, si tel est le cas, leur rapport est celui d'un nombre cube à un nombre cube (Él. VIII 27). Mais cette notion n'est pas utilisée ensuite dans la classification des irrationnelles.

38. In Nicom. arithm. introd. liber, 82.10-12 Pistelli (trad. N. Vinel). 
la forme pour les rendre semblables, tout en conservant leurs grandeurs mais, parmi les quatre côtés des nouveaux rectangles ainsi produits, il y en aura toujours au moins un qui ne correspondra à aucun nombre.

- Inutiles, car la même impossibilité arithmétique était déjà identifiable (et tout à fait explicite) dans le contexte plus restreint du Théetète. Dans l'un et l'autre cas, des droites non commensurables avec l'unité de mesure sont produites, mais ni l'Épinomis, ni le Théetète, ni d'ailleurs aucun texte mathématique grec ancien ne mentionnent ce que les Modernes appellent «nombre irrationnel » et le détour par la similitude euclidienne entre nombres n'apporte rien de plus ici.

Certains interprètes ${ }^{39}$, cherchant à souligner la cohérence mathémati-

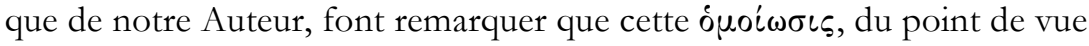
opératoire, revient à prendre une moyenne proportionnelle entre les nombres exprimant les côtés d'un nombre plan (ou deux moyennes proportionnelles entre l'unité et le nombre produit de trois facteurs, dans le cas d'un solide). Or la section (7) du témoignage fait elle aussi référence à l'intercalation de médiétés, non pas géométriques, mais arithmétique et harmonique ${ }^{40}$, dans la lignée du Timée, 35b4-36b6. Il faudrait donc com-

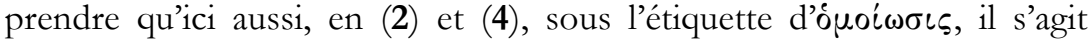
d'intercaler des moyennes (géométriques) et en conséquence, dans le cas solide (4), cela revient à percevoir une fine allusion au célèbre problème délien (duplication du cube). Van der Waerden va au bout de cette logique en soulignant que les mathématiques dont il est question dans l'Épinomis sont celles d'Archytas, célèbre pour ses travaux sur les moyennes ${ }^{41}$.

Cela dit, la considération de moyennes géométriques ne requiert pas non plus l'utilisation de la notion euclidienne de nombres semblables. Déjà, dans le contexte du Théétète, il s'agit de faire la quadrature d'une aire rectangulaire, et comme le dit Aristote, dans ce cas, faire une quadrature, c'est prendre une moyenne proportionnelle ${ }^{42}$. Il n'y a là rien d'innovant car cette propriété était connue bien avant Théetète ou Platon.

39. Notamment Jones 1932, p. 63-65 et Tarán 1975, p. 330-331.

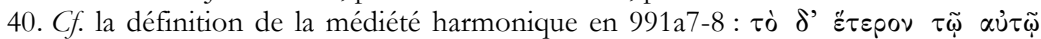

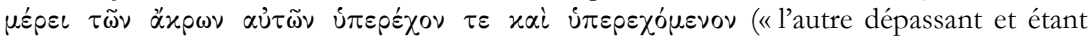

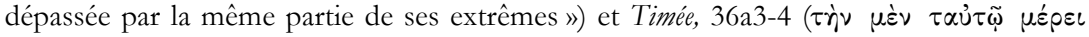

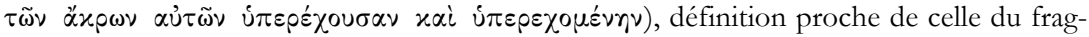

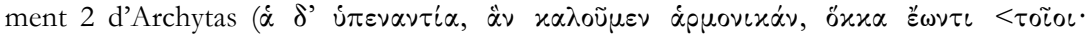

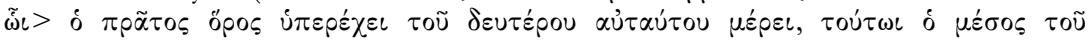

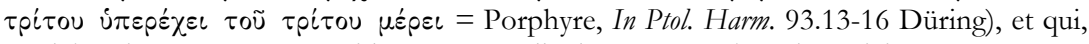
mathématiquement, ne coïncide pas avec celle des auteurs néopythagoriciens.

41. Van der Waerden 1954, p. 155. Cf. DK 47 B 2 et DK 47 A 14-15.

42. De anima, II 2, 413a13-20 et Metaph. B, 2, 996b18-21. 
Hippocrate de Chio maîtrisait suffisamment ces techniques pour opérer la réduction du problème délien à celui de l'insertion de deux moyennes proportionnelles, problème autrement complexe. En outre, la variation de l'expression (" moyenne(s)» au lieu de « similitude ») ne change rien à la situation mathématique: la prise d'une moyenne proportionnelle ne devient une opération universelle que lorsqu'on la pratique dans le cadre de la géométrie. Pour deux nombres entiers, elle n'est pas toujours possible (il faut et il suffit qu'ils soient semblables !). La supériorité de l'arithmétique, en ce domaine du moins, ne vaut pas, et ceci ne va pas du tout dans le sens du projet unitaire et pan-arithmétique de l'Auteur de l'Épinomis qui

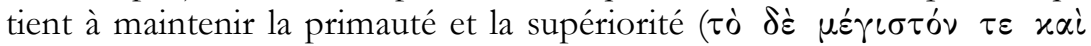
$\pi \rho \tilde{\omega} \tau o \nu, 990 c 5-6)$ de l'arithmétique. Enfin, si c'était ce qu'il avait voulu dire, il était bien facile d'expliciter ce lien entre médiétés et géométrie.

Ce n'est pas le cas : ce sont les exégètes qui croient dire bien ce que l'Auteur a mal dit! La cohérence ainsi produite se trouve, non pas dans le texte analysé, mais dans les contextes que l'on est susceptible de mobiliser pour son interprétation: les loci mathematici platoniciens authentiques, la littérature mathématique conservée, les indications générales que nous possédons sur l'histoire préeuclidienne des mathématiques... quitte à admettre l'imprécision du vocabulaire, la corruption textuelle au nom d'une sorte de "principe de charité » pour sauver un texte difficile. La démarche est en soi assez naturelle mais, dans ce cas, nous la croyons inappropriée.

\section{L’obscure reprise du Timée}

Si nous regardons rapidement la section (5-7), qu'observons-nous ? La formulation en est certes merveilleusement obscure, le texte assez incertain, mais le contenu mathématique est plutôt trivial. Même Taylor, chantre des mathématiques de l'Épinomis, n'y trouve que des considérations très élémentaires :

The meaning of the rest is that the succession of the "powers" $2^{1}, 2^{2}, 2^{3}$, is the most elementary example of the principle that similar areas have the duplicate and similar volumes the triplicate ratios of the corresponding "sides", and that the ratios corresponding to the fourth and fifth in the scale, respectively the sं $\pi$ i $\tau$ peros and

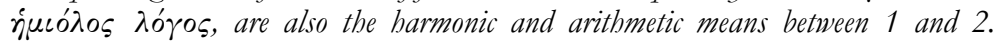
(Plato selects 6 and 12 as surrogates for 1 and 2 in this illustration because he wishes the two "means" to be whole numbers $)^{43}$.

43. Taylor 1926, p. 501, suite de la note 1, partiellement citée plus haut, note 29. Remarquez la parenthèse : Taylor est un partisan de l'authenticité ; Platon, dans l'Épinomis, aurait été le premier (et le seul) des Grecs à concevoir les nombres irrationnels, 
Le passage mathématique conjuguerait donc une revendication inouïe concernant les irrationnelles dans sa première partie et un lieu commun très élémentaire, à propos des médiétés, dans la seconde. Il y a là, sinon une contradiction, du moins une étrangeté. En fait cette seconde partie ressemble à un patchwork d'expressions et de citations, éventuellement tronquées, reprises pour l'essentiel au Timée ${ }^{44}$. Nous allons voir que cette dépendance lexicale et terminologique de notre passage vis-à-vis du dialogue cosmologique de Platon prévaut encore dans la cinquième et dernière occurrence des mathématiques en 991d8-992a1.

Pour conclure notre analyse de cette portion, disons que le mode de composition perceptible dans la section (5-7) - patchwork d'expressions et de notions mathématiques reprises aux dialogues de Platon -, vaut pour l'ensemble du passage mathématique, y compris celui sur la géométrie (2)-(4) pour lequel les modèles, plus ou moins bien compris, ont probablement été les loci mathematici du Théétète (147d4-148b8) et du Livre VIII de la République (546b4-c7) ${ }^{45}$. Il s'agit des seuls passages des dialogues où un usage conjoint de l'arithmétique et de la géométrie est mis en œuvre, ce qui pouvait servir le propos «unifiant» de notre auteur. À l'inverse, malgré son usage extensif du Timée, il s'est abstenu d'introduire quelque développement que ce soit concernant les polyèdres réguliers, hormis une brève mention et, dans sa description de l'astronomie, il s'est contenté de mentionner les huit périodes célestes - système numérique mais pas le système simple à double sphère que Platon décrit en Timée, 36b7-d7, 38d5-39b2, et qui rend (partiellement) compte, par la géométrie, du mouvement apparent des astres.

mais il se serait cru obligé de raisonner en nombres entiers pour les moyennes arithmétique et harmonique!

44. Outre la quasi citation déjà évoquée pour définir la médiété harmonique, on

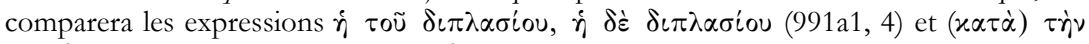

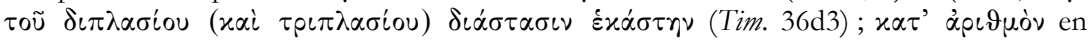

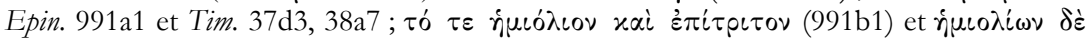

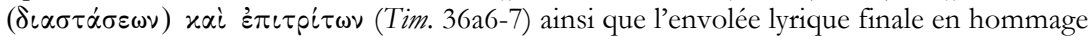
au chœur des Muses (991b2-4) avec la justification du don divin que constituent la voix,

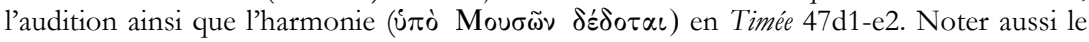

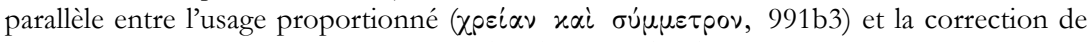
notre tendance au défaut de mesure ( nous offrent.

45. Que l'Auteur de l'Épinomis s'inspire du célèbre et obscur passage sur le nombre nuptial peut trouver confirmation dans l'allusion audit passage, déjà détectable en 977e5-

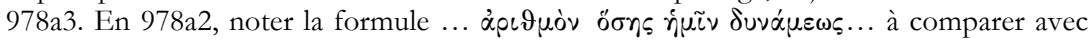
990c7-8. 


\begin{tabular}{|c|c|}
\hline 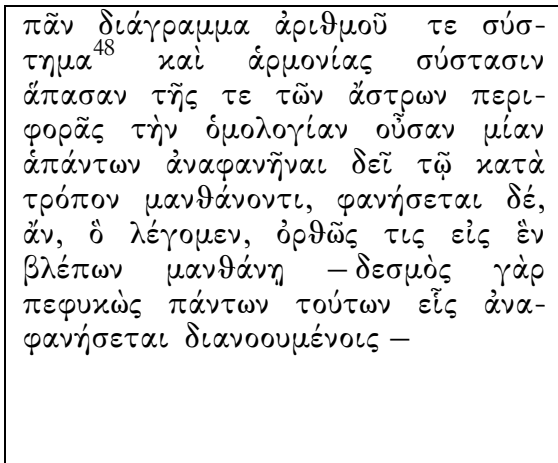 & $\begin{array}{l}\text { Tout diagramme et toute combinaison } \\
\text { de nombre et toute construction } \\
\text { d'harmonie, y compris celle des révo- } \\
\text { lutions sidérales, devront manifester } \\
\text { l'homologie, commune à toutes et qui } \\
\text { est unique, à celui qui étudie selon la } \\
\text { bonne manière et qui se manifestera, } \\
\text { comme nous le disons, si quelqu'un } \\
\text { étudie correctement en fixant son } \\
\text { regard sur l'un - car un lien, commun } \\
\text { par nature à toutes ces choses, unique, } \\
\text { apparaîtra à ceux qui réfléchissent. }\end{array}$ \\
\hline
\end{tabular}

Proclus, comme Théon et Jamblique avant lui, lit clairement ce passage comme traitant de l'àva $\lambda_{0 \gamma} \hat{\gamma}^{\alpha} \alpha$ là où l'original semble avoir porté

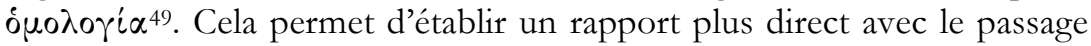
"mathématique » qui précède, dont la dernière partie était occupée à la question des médiétés (l'un des emplois mathématiques du terme $\alpha$ $\nu \alpha \lambda 0$ $\gamma^{\prime}(\alpha)$. Proclus va donc s'opposer très clairement à l'idée que l'àva $\lambda \gamma_{0} \gamma^{i} \alpha$ puisse être considérée comme ce qui donne son unité aux différents

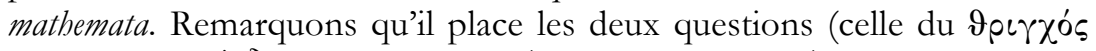
et celle du $\sigma u ́ v \delta \varepsilon \sigma \mu \circ \varsigma$ ) sur le même plan alors même qu'il conteste l'authenticité de l'Épinomis. Cela indique, comme le confirmera l'examen des sources antérieures, que la question de ce qui fait le «lien » des différentes sciences mathématiques était alors devenue une question classique

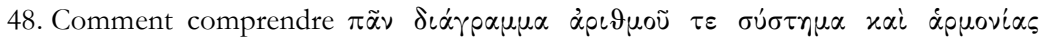

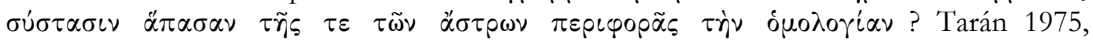

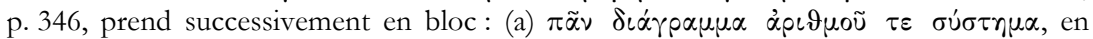
considérant que $\delta \iota \alpha ́ \gamma p \alpha \mu \mu \alpha$ se rapporterait au nombre plan, $\sigma \dot{\sigma} \sigma \tau \mu \alpha$ au nombre solide

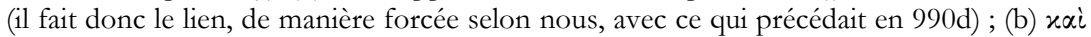

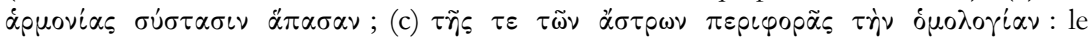

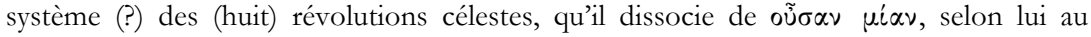

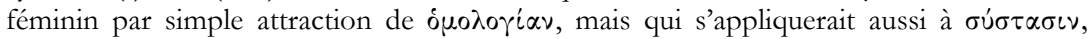

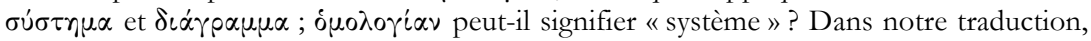
la récapitulation correspond au quadrivium classique ; dans l'interprétation de Tarán (malheureusement il ne donne pas de traduction), il n'y aurait que trois sciences: celle du nombre (correspondant à l'arithmétique et à la géométrie, pour le coup réduite à un rôle de représentation figurée de données arithmétiques), l'harmonique et l'astronomie. On pourrait même les réduire à deux : la science des nombres et celle de l'harmonie astronomico-musicale, l'étude de la première étant préalablement requise pour la pleine compréhension de la seconde. Là encore la cohérence ainsi produite est externe au texte et la grammaire ne semble pas favoriser cette lecture.

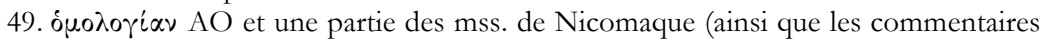
d'Asclépius et de Philopon), à $\alpha \lambda \circ \gamma^{\prime} \alpha \nu$ l'autre partie des mss. de Nicomaque, de Théon et de Jamblique. 
dans ce type d'exposé (au même titre que celle de leur utilité, de leur but ou de leur objet). Pour autant, ces deux questions ne sont évidemment pas traitées sur un pied de parfaite égalité puisqu'il s'agit dans un cas de justifier la réponse de Platon (la dialectique comme couronnement des mathemata), et dans l'autre de contester une réponse jugée non platonicienne. Cette réponse n'est pas référée seulement à l'Épinomis, la discussion s'ouvrant par l'exclamation suivante :

Quant au lien des mathématiques, nous ne devons pas le poser dans l'à $\alpha \lambda$ ớ $\alpha$ comme le pense Ératosthène. (In Eucl. 43.22-23 Friedlein.)

Le couplage entre la doctrine de l'Épinomis et les réflexions d'Ératosthène n'est pas propre à Proclus puisqu'il est attesté chez Théon de Smyrne, qui cite à ce propos le dialogue perdu d'Ératosthène intitulé $\Pi \lambda \alpha \tau \omega \nu \iota x o ́ s$. Avant d'entrer dans cette confrontation, il convient toutefois de faire encore quelques remarques sur la position du problème telle qu'elle apparaît chez Proclus. La réponse du Lycien à ce qu'il identifie comme la thèse d'Épinomis-Ératosthène consiste, pour l'essentiel, à faire valoir sa propre vision de ce qui fait l'unité des mathématiques au titre de la notion, brièvement développée dans les chapitres précédents, de

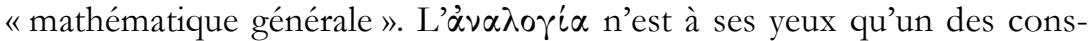
tituants de cette ö $\lambda \eta \mu \alpha \vartheta \eta \mu \alpha \tau \iota x \dot{\eta}$ et il n'est donc pas légitime d'en faire l'unique «lien» des mathemata. Ce refus provient de la claire conscience qu'a Proclus des difficultés liées au fait d'exhiber une théorie générale et unitaire dans les mathématiques qu'il commente (les Éléments d'Euclide), surtout en ce qui concerne le rôle des proportions. En découle une caractérisation à la fois plus large, et aussi plus philosophique, des contours de ce qui peut faire lien entre les différentes disciplines mathématiques ${ }^{50}$.

Cela dévoile, par contrecoup, un certain nombre de problèmes qu'il faut avoir en mémoire pour comprendre les débats liés à la réception antique de l'Épinomis. Il est essentiel, tout d'abord, de constater que chez les auteurs que Proclus critique ici - et dont nous verrons qu'outre Théon, ils comptent Nicomaque de Gérase et Jamblique de Chalcis - le modèle euclidien que Proclus cherche à mettre en avant est absent. Cela peut paraître curieux aux yeux d'un lecteur moderne qui a tendance à considérer que le cœur des mathématiques grecques anciennes est donné par la triade Euclide-Archimède-Apollonius, et que le cœur de ce cœur est la «théorie des proportions » qui forme le noyau opératoire de leurs mathématiques. Même si l'on peut aisément comprendre que des auteurs d'inspiration néopythagoricienne du second ou du troisième siècle de 
notre ère privilégient d'autres références, on peine à comprendre qu'ils ne prennent même pas la peine de discuter cette position alternative de

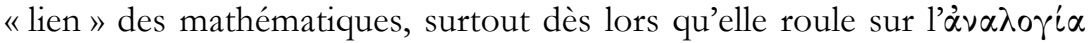
dont un des sens est précisément « proportion ».

Mais l'on peut aussi prendre appui sur ce constat pour mettre en question une évidence peut-être trompeuse : comme en témoignent les méandres de la construction de Proclus, trouver dans les mathématiques un «lien »-au sens d'une position forte d'unité - est loin d'être chose évidente, surtout dès lors qu'on constate que cette mathématique sépare d'emblée avec soin nombres et grandeurs (élaborant en conséquence non pas une, mais deux théories des proportions, comme on peut en trouver aux livres V et VII des Éléments d'Euclide). Or cette position d'unité n'en semble pas moins exigée par certains programmes philosophiques, comme celui proposé par Platon lorsqu'il demande de l'apprenti philosophe, dans un passage célèbre de la République, qu'il saisisse la commu-

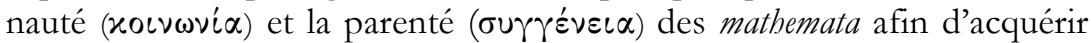

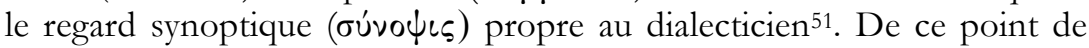
vue, le détour par Proclus est utile pour comprendre non seulement le contexte dans lequel le passage mathématique de l'Épinomis a pu se trouver utilisé, mais aussi les raisons qui en faisaient, en un sens, la matrice d'une «solution » idéale à un problème (philosophique) que les théories (mathématiques) ne semblaient pas pouvoir régler d'elles-mêmes. Ce cadre est très différent de celui que projettent les commentateurs récents lorsqu'ils lisent ce texte comme un témoignage sur une théorie mathématique puissante cachée sous une rhétorique philosophique qu'ils auraient à charge de déchiffrer.

\section{L'Épinomis chez Théon, Nicomaque et Jamblique}

Comme indiqué précédemment, le couplage du passage mathématique de l'Épinomis et des conceptions d'Ératosthène se trouve déjà dans l'Expositio de Théon de Smyrne, qui est l'auteur antique qui nous donne le plus d'indications sur l'un et sur l'autre. Le début de son «Exposé des connaissances utiles à la lecture de Platon » est tout à fait exemplaire du rôle qu'on pouvait alors faire jouer à l'Épinomis pour reconstruire un programme platonicien homogène et compatible avec les présupposés

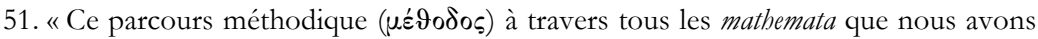
exposés, s'il parvient à ce point où ils trouvent les uns par rapport aux autres leur communauté ( ils sont apparentés les uns aux autres, en tirera quelque contribution en vue de cette recherche que nous souhaitons mener, et ce ne sera pas en vain que nous aurons peiné.» (Resp. VII, 531c9-d3 ; trad. Leroux 2002 modifiée.) Sur le « regard synoptique », cf. Resp. $537 \mathrm{c}-\mathrm{d}$. 
des auteurs néopythagoriciens. Toute l'ouverture du traité consiste, en effet, en une juxtaposition de citations tirées de la République de Platon (du livre VII bien sûr, mais aussi du livre III) sur le rôle des mathématiques dans l'apprentissage philosophique, entremêlées de longs extraits de l'Épinomis. Ce montage infléchit sensiblement le programme de la République en posant beaucoup plus clairement la primauté de l'arithmétique comme noyau unificateur des mathématiques et en laissant à la dialectique une portion congrue (l'insistance étant plutôt mise dans l'Épinomis, comme nous l'avons vu, sur le rôle de l'«astronomie» ou, pour être plus exact, d'une théogonie et d'une cosmogonie, soutiens de la vertu et de la piété du sage). C'est dans le contexte de cette introduction que Théon cite le début du passage «mathématique » (9.12-10.11 Hiller, reprenant Epin. 990c2-e1) où est clairement posée la préséance de l'arithmétique, suivie de cette discipline qu'on appelle du «nom tout à fait

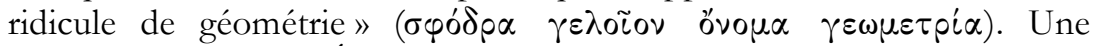
première référence à Ératosthène était alors apparue, déjà couplée à une mention de Epin. 992a-b, mais elle se limitait à l'anecdote célèbre sur la duplication de l'autel exigée par l'oracle auprès des Déliens, oracle dont Platon aurait estimé qu'il avait été prononcé pour signifier aux Grecs leur négligence des mathématiques (Expositio, 2.3-12 Hiller).

Des indications plus nourries sur le contexte dans lequel a pu se développer le programme contre lequel se dresse Proclus sont données dans la seconde partie du traité, dévolue aux théories musicales. C'est en

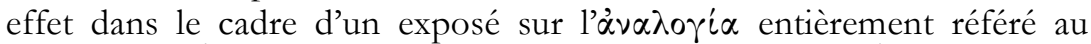
Platonicien d'Ératosthène qu'est cité notre passage de l'Épinomis sur le «lien» ( $\delta \varepsilon \sigma \mu o ́ \varsigma, 84.13$ Hiller). Le contexte est celui d'une réflexion géné-

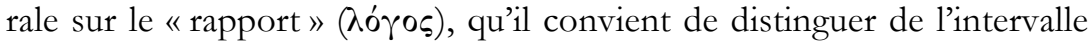
$(\delta \iota \alpha ́ \sigma \tau \eta \alpha)$ et qui est présenté dans le cadre d'une réflexion philosophique générale sur les «éléments » des mathématiques. Le rapport d'égalité est alors présenté comme "principe» et "cause première » de

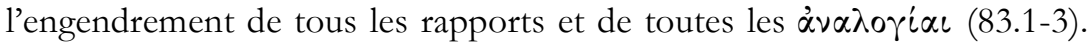
Théon enchaîne ensuite sur le thème de la cohésion des mathématiques

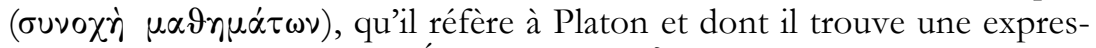
sion dans le passage de l'Épinomis sur le $\delta \varepsilon \sigma \mu o ́ s$ (avec le changement dont semble hériter Proclus de l'ó $\mu \circ \lambda \circ \gamma^{\prime} \propto \alpha$ en $\left.\alpha \nu \alpha \lambda o \gamma i \alpha\right)$. Dans la suite du traité ( $(51)$, il revient longuement sur la position de " principe » que peut tenir le "rapport d'égalité » en expliquant comment on peut engendrer, à partir d'une suite de trois termes égaux, les différents rapports numériques par une procédure connue aujourd'hui sous le nom de 
« règle d'Adraste » (parce que Théon attribue à ce dernier le fait d'avoir « démontré » ce qu'Ératosthène n'avait fait que « montrer » $)^{52}$.

Ce passage peut être mis en parallèle avec les développements de Pappus au livre III de sa «Collection $»^{53}$, dans une section consacrée à la théorie des médiétés. Même si l'Épinomis et Ératosthène ne sont pas cités, il est tout fait remarquable que Pappus (ou un de ses commentateurs) ait

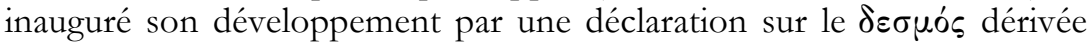
$\mathrm{du}$ « divin Platon ${ }^{54}$. Par ailleurs, ce rapprochement permet de comprendre pourquoi Théon (mais aussi bien Nicomaque qui décrit une procédure semblable) expose l'engendrement des rapports à partir d'une suite de trois nombres, là où deux auraient pu suffire. De fait, Pappus propose une procédure permettant d'engendrer à partir de la raison d'égalité non seulement les différentes formes de rapports numériques, mais toutes les médiétés. Ce second engendrement se fait à partir de la médiété géométrique obtenue facilement par le premier procédé, ce qui semble indiquer un possible contexte général pour les réflexions autour de l'à $\alpha \lambda \gamma_{0} \gamma^{\prime} \alpha$ comme lien des mathemata ${ }^{55}$.

La chose est d'importance, car à s'en tenir au seul témoignage de Théon, on pourrait objecter que les passages qu'il attribue au Platonicien et le programme de l'Épinomis ne sont pas aussi concordants qu'il y parait. Juste après avoir cité le passage sur le $\delta \varepsilon \sigma \mu o ́ s$, Théon reprend, en effet, le fil de son exposé sur les rapports en insistant sur la distinction à faire

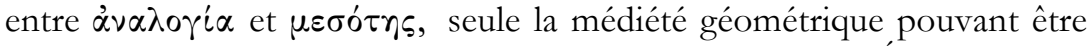
proprement qualifiée d'à $\alpha \lambda \lambda_{0} \gamma_{i} \alpha$. Or si le passage de l'Épinomis com-

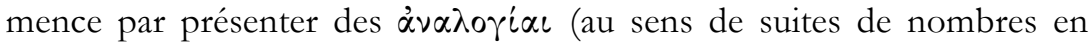
proportion continue), c'est pour mieux passer ensuite à la considération des différentes moyennes ( $\mu \varepsilon ́ \sigma \alpha \iota)$ dans un passage clairement décalqué du fameux passage de Timée, 35b4-36b5, sur les deux médiétés ("arithmétique » et "harmonique »). Lorsque les commentateurs récri-

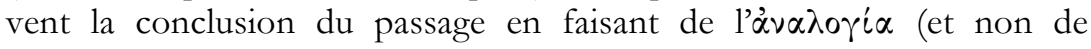

52. Expositio, 107.23-24 Hiller. Pour une description de cette procédure, voir Vitrac 2008a, p. 94-97.

53. Collectio, 68.17-104.13 Hultsch.

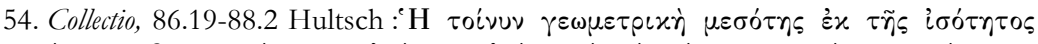

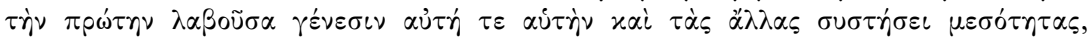

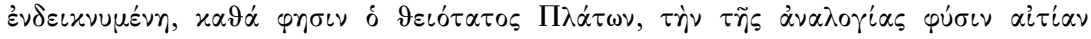

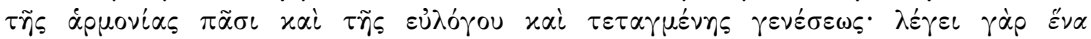

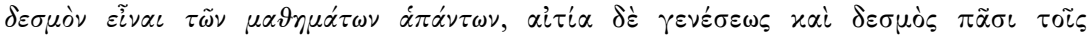

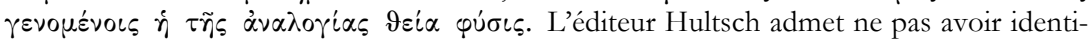
fié la source platonicienne de cette prétendue citation et suggère qu'il s'agit d'une compi-

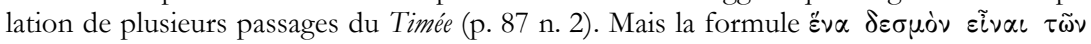
$\mu \alpha 9 \eta \mu \alpha \dot{\tau} \tau \omega \nu \dot{\alpha} \pi \dot{\alpha} \nu \tau \omega \nu$ est clairement une interprétation d'Epin. 991e3-992a2.

55. Vitrac 2008a, p. 97. 


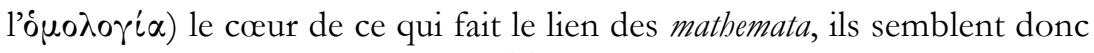
pratiquer l'amalgame que critique Théon en renvoyant à un exposé où se sont succédé progressions de nombres en proportion continue et descriptions des médiétés (doublé certainement d'un jeu de mots sur le sens

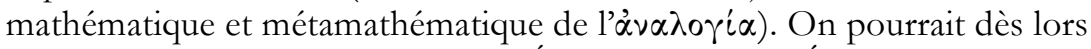
se demander si le programme de l'Épinomis et celui d'Ératosthène étaient vraiment compatibles et si leur jonction ne relève pas purement et simplement d'un malentendu. Le passage de Pappus indique qu'on n'est nullement forcé à cette conclusion et que l'on pouvait tout à fait, dans le sillage d'un procédé effectivement mis en avant par Ératosthène, consi-

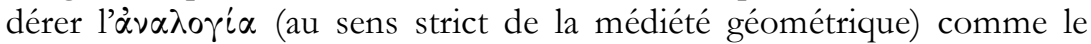
principe d'engendrement de toutes les médiétés, si bien qu'elle était conformément au texte de Timée, 31c, qui sert de toile de fond à ces discussions - le vrai «lien» de toutes choses (le passage sur les médiétés de l'Épinomis se concluant sur le fait qu'elles donnent aux hommes « un usage consonant et proportionné en vue d'un jeu de rythme et d'harmonie, offrande au chœur bienheureux des Muses »).

On ne retrouve pas chez Nicomaque, ni chez Jamblique, une telle cohérence entre les références à l'Épinomis et à la théorie mathématique des

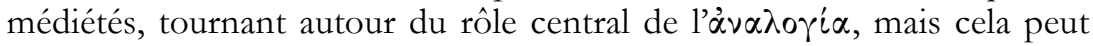
aisément s'expliquer dans la mesure où Nicomaque refuse précisément l'idée que la proportion géométrique serait le cœur de la théorie des médiétés et insiste régulièrement sur la primauté de l'arithmétique, d'où celle de la médiété homonyme sur celles qui sont homonymes des autres sciences ${ }^{56}$. Il n'en reste pas moins qu'on va retrouver au premier rang, chez l'un comme chez l'autre, le passage sur le «lien» des mathématiques (chose d'autant plus remarquable que les références à Platon se font chez eux plus rares que chez Théon).

Chez Nicomaque, le passage est mentionné dès le début du traité pour appuyer l'idée que les sciences mathématiques nous ménagent un accès aux formes primordiales d'être et conditionnent donc l'accès à la sagesse. Il est à noter que le passage est alors présenté comme appartenant au «treizième livre des Lois, intitulé par certains Le philosophe» (I,

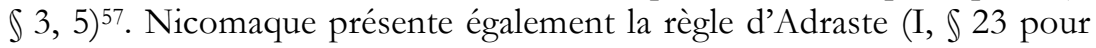
les méthodes directes) qu'il complète d'ailleurs par l'exposé de la procédure inverse (réduction à l'égalité, II, $\$ 1-2$ ), mais sans mentionner

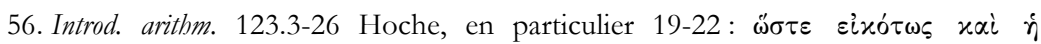

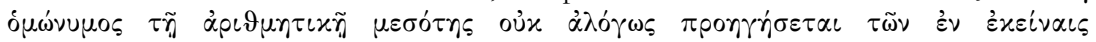

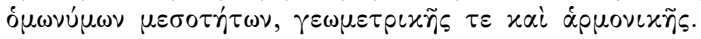

57. Introd. arithm. 7.4-6 Hoche. 
l'Épinomis, ni Ératosthène, ni Adraste, ni quelque source que ce soit ${ }^{58}$. Cela dit, il est remarquable qu'il précise à cette occasion qu'« une telle théorie a pour conséquence un théorème tout à fait remarquable et utile pour la psychogonie platonicienne, ainsi que pour tous les intervalles harmoniques » (75.14-17 Hoche), référence transparente au rôle que pouvaient tenir à ses yeux ces techniques dans la compréhension du Timée.

Chez Jamblique, le passage sur le «lien » est repris tel quel au début du chapitre 6 du traité "Sur la science mathématique commune», mais sans référence à Platon ni à l'Épinomis. Le chapitre suivant reprend néanmoins la discussion dans une référence explicite à Platon et dans un montage avec les thèmes de République VII sur la "parenté » et la «communauté » des mathemata, dans l'esprit de ce qu'avait fait Théon, quoique de manière beaucoup plus condensée ${ }^{59}$. Une séquence comparable apparait dans son commentaire de Nicomaque où il ajoute à nouveau

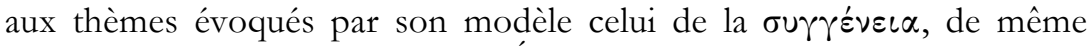
qu'il décalque les passages de l'Épinomis sur la mathématique comme condition de l'accès à la plus haute sagesse (In Nic. 9.5-14 Pistelli). La piste ouverte par les critiques de Proclus s'avère donc très fructueuse : chez tous nos auteurs, on retrouve parmi les questions préjudicielles celle de l'unité et de la communauté des mathemata et c'est dans ce contexte que se situe clairement leur reprise de l'Épinomis.

\section{Relire le passage mathématique de l'Épinomis à partir de la question du «lien »}

Ce rapide descriptif des lectures anciennes du passage mathématique de l'Épinomis nous donne deux indications importantes. Tout d'abord, il est pour le moins significatif qu'aucun commentateur ancien, certains

58. Il faut d'ailleurs souligner que cette présentation se trouve, non pas dans sa quatrième partie consacrée aux médiétés, mais dans la deuxième qui traite de l'analyse de la quotité en relation (classification des rapports numériques).

59. Comm. Math. VII, 31.4-17 Festa : « Puisque leurs objets sont apparentés ( $\alpha \dot{\delta} \varepsilon \lambda \varphi \dot{\alpha})$, il est raisonnable de considérer également ces sciences comme étant apparentées (si l'on ne veut pas prêter des puérilités à cette phrase d'Archytas : "car ces sciences mathématiques semblent être apparentées") et, comme elles sont reliées l'une à l'autre, de les considérer comme une sorte de chaîne formée d'anneaux; et elles se terminent par un lien

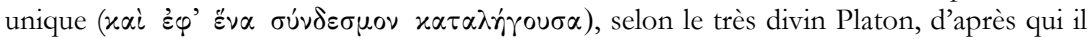
conviendrait que soit unique la parenté ( $\sigma u \gamma \gamma \varepsilon \dot{v} \varepsilon\llcorner\alpha \nu)$ qui se révèle entre ces sciences

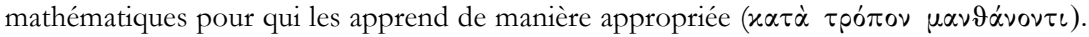
Mais celui qui, comme Platon le suppose lui-même, a saisi ainsi tout l'ensemble de ces sciences mathématiques, Platon l'appelle en effet l'homme parfaitement sage, au sens le plus véritable, et il l'affirme sur un ton plaisant; il recommande à ceux qui s'adonnent avec empressement à la philosophie de se mettre en quête de ces sciences mathématiques et de les préférer systématiquement, qu'elles soient difficiles ou faciles. " (Traduction Gérald Bechtle, communication personnelle.) 
pourtant prompts à attribuer à Platon les plus grandes capacités mathématiques, n'ait vu dans le texte la moindre allusion à une théorie mathématique de l'irrationalité. De fait, ce texte leur semble relever d'une tout autre problématique : celle de l'unité des mathématiques et de la thèse selon laquelle le «lien» des différentes mathématiques serait à trouver dans $l^{\prime} \alpha \dot{\nu} \alpha \lambda \circ \gamma^{\prime} \alpha^{60}$. Qu'on interprète cette $\alpha^{2} \nu \alpha \lambda o \gamma^{\prime} \alpha$ au sens strict de proportion géométrique (et selon la procédure d'engendrement décrite par Théon et Pappus permettant, à partir d'elle, d'obtenir toutes les médiétés), ou directement au sens large de médiété, ces deux interprétations s'accordent avec le passage de l'Épinomis. C'est la raison pour laquelle nous n'avons pas traduit un terme qui pourrait bien avoir, selon l'auteur qui cite le texte, un sens différent.

Cette lecture permet de donner au passage une cohérence philosophique qui, sans cette clé, paraît pour le moins difficile à saisir (car nous avons vu dans les parties précédentes à quel point la cohérence mathématique sous-jacente parait pour le moins incertaine). Imaginons, en effet, un lecteur de Platon qui chercherait à rassembler en une vue synthétique la formation du philosophe et les conditions censées le mener à la plus haute sagesse. Partant du programme de République VII et prenant acte du rôle que doivent tenir dans ce curriculum les mathématiques, il se heurterait immédiatement à la question de savoir si la "parenté » et la «communauté » nécessaires à l'acquisition de la vue synoptique que le dialecticien porte sur l'ensemble du savoir sont le fait d'un regard extérieur sur les mathemata, ou si elles correspondent à une réalité donnée de manière intrinsèque à ces disciplines. Il lui faudrait donc chercher dans d'autres lieux du corpus des éléments précisant l'articulation entre cette « communauté » des mathématiques et la vue synthétique du philosophe - tâche d'autant plus importante qu'on refuse, avec les auteurs que nous avons croisés jusqu'à présent, de couper la dialectique des mathématiques $^{61}$. Or ces lieux ne sont pas si nombreux.

L'un d'entre eux était assurément donné par le Philèbe (16c-17a), où Socrate décrit la «voie » (ódós) dont il est amoureux et de laquelle, dit-il, tous les arts ont tiré le principe de leurs découvertes. Suit alors la fameuse référence aux «Anciens » censés avoir transmis une doctrine dont

60. C'est aussi dans ce contexte que Cicéron reçoit le passage, notamment dans $D e$ oratore, III, 5, 19-6, 21, où il s'appuie sur Platon pour établir que la connaissance repose sur le «lien» (vinculum) reliant entre eux les arts humains dans une même communauté (societas).

61. Jamblique consacre un long développement à cette question dans son De Com. Math. Sc. (\$ 27-29). Il semble alors s'opposer à d'autres néoplatoniciens (Porphyre ?) qui séparaient plus nettement, dans l'héritage d'Aristote, le niveau logique et le niveau mathématique. 
les néoplatoniciens se nourriront abondamment: «tout ce qu'on peut dire exister est fait d'un et de multiple, et contient en soi-même, origi-

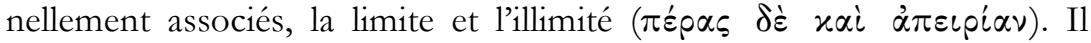
nous faut donc, puisque les choses sont ainsi ordonnées, poser toujours, en quelque ensemble que ce soit, et chercher en chaque cas une forme

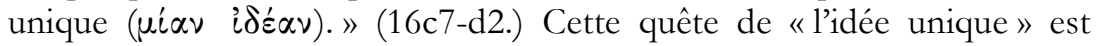
alors présentée sur le modèle de la recherche de nombres à insérer dans des intervalles :

Si donc nous l'appréhendons, après cette unique forme examiner s'il y en a deux, et, sinon deux seulement, trois ou quelque autre nombre; puis faire le même examen pour chacune de ces unités nouvelles, jusqu’à ce que, de cet un primitif, on voie non seulement qu'il est un et multiple et illimité, mais aussi quelle quantité précise il atteint ; quant à la forme de l'illimité, ne pas l'appliquer à la multitude avant d'avoir saisi quel nombre total celle-ci réalise dans l'intervalle entre l'illimité et l'un; alors seulement laisser chacune des unités de cet ensemble se disperser dans l'illimité. » (16d2-e2, trad. A. Diès modifiée.)

Un autre texte que pouvait mobiliser un lecteur de Platon pour donner plus de consistance au programme d'une unité des mathemata est évidemment Timée, 35b4-36b5. Le rapprochement avec le passage qui précède était d'ailleurs d'autant plus naturel qu'un des objets du Philèbe, développé dans la suite de l'exposé, est précisément de donner un sens à la notion de «mélange ». Il permettait surtout de donner un sens beaucoup plus précis à l'impératif d'une recherche du «nombre» dans les «intermédiaires", en mobilisant cette fois directement la théorie des rapports numériques constitutifs de l'âme du monde (et donc, pour nombre d'interprètes de Platon, de toute âme). On se rappelle en effet que le démiurge, ayant "mélangé » l'indivisible et le divisible, en tirait la substance intermédiaire de l'âme du monde, dont la forme apparaissait ainsi comme unifiant le Même et l'Autre (35a). Il procédait ensuite à la division de ce tout selon certains rapports (les premières relations invoquées étant les multiples). Une deuxième étape consistait alors à « combler les intervalles doubles et triples » (c'est-à-dire les intervalles de la suite des nombres en progression géométrique de raison 2 et $3: 1,2,3$,

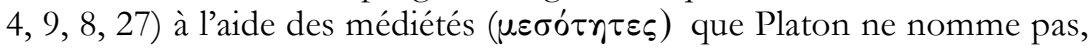
mais que la tradition, dès le fragment DK 47 B 2 d'Archytas, appelle «arithmétique» et «harmonique » ${ }^{62}$. Cette seconde opération donnait

62. A noter que lorsque l'auteur de l'Épinomis démarque ce passage, il mentionne seulement la progression selon la raison double, ce qui nous faisait parler plus haut de «citations parfois tronquées ». 
naissance à une nouvelle série de rapports (caractéristique de la gamme diatonique : hémiole, épitrite, épogde) censée manifester l'harmonie du monde.

Or nombre de lecteurs opèreront - à tort - un rapprochement entre

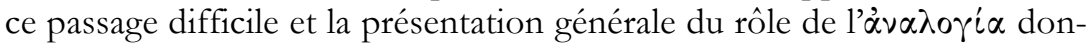
née en 31 c à propos du «lien» du feu et de la terre :

Mais que deux termes forment à eux seuls une belle composition sans un troisième, cela n'est pas possible. Quelque lien ( $\delta \varepsilon \sigma u \dot{\nu} \nu)$ intermédiaire doit naitre pour les rapprocher l'un de l'autre. Or, de tous les liens, le plus beau est celui qui de lui-même et des termes qu'il relie crée l'unité la plus forte. Et cela c'est la proportion (å $\vee \alpha \lambda o \gamma i \alpha)$ qui naturellement le réalise de la façon la plus belle. Lorsque de trois nombres, ou masses, ou puissances quelconques ${ }^{63}$, le moyen est tel que ce que le premier est par rapport à lui, ce moyen lui-même le soit par rapport au dernier ; et inversement, quand il est tel que, ce que le dernier est par rapport au moyen, le moyen le soit par rapport au premier, le moyen devenant alors à la fois le premier et le dernier, le premier et le dernier devenant tous deux moyens à leur tour, il arrive ainsi nécessairement que tous les termes aient la même fonction, que tous jouent les uns par rapport aux autres le même rôle, et dans ce cas tous forment une unité parfaite... ${ }^{64}$ Par ces procédés et à l'aide de ces corps ainsi définis et au nombre de quatre, a été engendré le corps du Monde. Accordé par la proportion ( $\delta \iota^{\prime}$ àva $\lambda_{0-}$

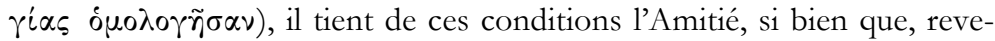
nant sur lui-même en un seul et même tout, il a pu naître indissoluble par toute autre puissance que celle qui l'a uni. (Tim. 31b8-32a7, 32b9-c4, trad. A. Rivaud modifiée.)

Ces passages sont clairement en arrière-fond du passage mathématique de l'Épinomis qui d'un côté décalque le développement sur les moyennes, de l'autre se conclut sur le «lien» de toutes choses (les lecteurs tardifs ne pouvant s'empêcher de lire alors àva $\lambda_{0} \gamma^{\prime} \alpha$ à la place

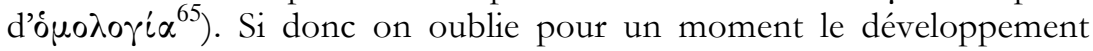
pour le moins obscur sur la géométrie - passage qui a focalisé l'attention des lecteurs modernes au point peut-être de leur faire perdre de vue l'unité thématique du passage - on voit que le reste du texte correspond

63. Pour la signification de ce passage et le choix de traduction qui en découle, voyez Vinel 2003.

64. Ici s'insère le passage sur le nombre de médiétés requises dans les cas plan et solide, que nous avons cité supra n. 25.

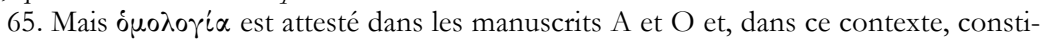
tue la lectio difficilior. Notre auteur, une fois encore, a brutalement résumé l'expression de

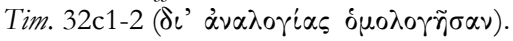


assez clairement à un collage destiné à faire tenir ces différentes indications dans un seul programme. Ce que propose a minima le passage mathématique de l'Épinomis, d'après les commentateurs anciens, c'est une position d'unité des mathématiques donnée de l'intérieur de ces sciences

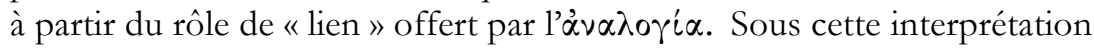
le passage mystérieux sur «l'assimilation » des nombres correspond alors très précisément à la difficulté qu'avait (et qu'a encore) n'importe quel lecteur qui veut forcer la cohérence de ces loci platoniciens, si bien qu'on se sent moins naturellement porté à y lire le témoignage de quelque théorie mathématique puissante, mais cachée. Ainsi n'y a-t-il aucune raison de considérer que le caractère contourné d'expressions apparemment dénuées de sens mathématique obvie («assimilation» des nombres non semblables) renvoie à quelque théorie cachée que l'interprète aurait à charge de reconstituer, et il paraît beaucoup plus probable qu'elle corresponde à une pirouette en un point particulièrement délicat du développement, dont tout laisse plutôt à penser que l'auteur ne maitrise pas les attendus techniques sous-jacents.

\section{Conclusion}

Aussi décevante que puisse paraitre une telle conclusion, la composition du passage dit «mathématique » de l'Épinomis semble, au bout du compte, relever essentiellement du collage, et qui pis est d'un collage mal fait : reprise d'expressions et de formules à différents loci mathematici platoniciens, citations tronquées, imitation du style qui ne fait que renforcer l'obscurité et l'imprécision. Il n'y a là aucune trace d'une théorie mathématique originale (pace Taylor), ni même de vaste récapitulation du savoir mathématique de l'époque platonicienne (pace des Places). Dans le contexte des mathématiques de l'époque classique qui a été témoin des premières grandes réussites de la géométrie grecque, l'orientation fondamentalement arithmétisante de l'Épinomis, la promotion de l'astronomie - d'une certaine approche de l'astronomie -, l'éloge appuyé du nombre comme élément ordonnant et harmonisant le cosmos, peuvent recevoir différentes interprétations. On peut y reconnaitre une lecture «pythagorisante » ("Tout est à l'imitation du nombre ») et sélective du Timée. Peut-être, par charité, pourrait-on également y percevoir l'influence des modèles astronomiques de type numérique d'origine babylonienne dont les Grecs prirent connaissance vers le milieu du IV siècle av. J.-C.

Quoi qu'il en soit, un des effets indéniables de cette présentation est de souligner l'exigence d'unité des mathématiques, même si, à s'en tenir à la lettre du texte, celle-ci semble décrite dans les termes d'une vague

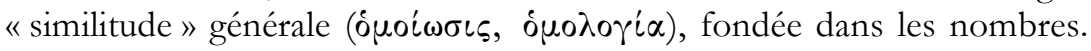


Mais le dialogue, dès l'époque hellénistique, était attribué à Platon et les exégètes ne pouvaient certainement pas se contenter d'une lecture aussi plate, d'autant que la question de l'unité des mathématiques, pour les courants philosophiques issus de son enseignement, était un vrai problème. Aussi firent-ils le rapprochement entre les propos unificateurs ${ }^{66}$ de la première partie du Timée et le passage de l'Épinomis qui, au demeurant, cite ce texte. Cependant, là où Platon recourait à différents éléments d'une même manipulation mathématique (l'insertion de médiétés) pour unifier d'une part le corps du monde (Tim. 31c), d'autre part l'Âme du monde (36a), ils crurent reconnaitre la proclamation d'une unité forte des mathématiques, de l'Âme et du corps du monde - qu'énonçait aussi à sa

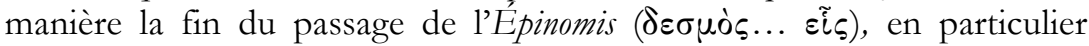

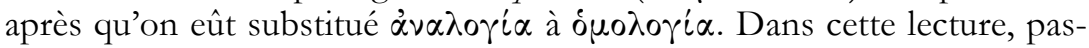
ser du niveau des objets mathématiques (diagramme, nombre, harmonie,

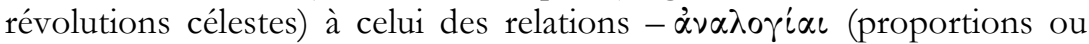
médiétés) - fournissait une réponse au problème de l'unité des mathématiques.

Même si, avec Proclus, on n'accepte pas cette réponse, on doit y reconnaître un acte métamathématique fort. Les trois médiétés «classiques » étaient considérés par les mathématiciens contemporains ${ }^{67}$ de Platon comme relevant d'une seule et même démarche mathématique. Bien avant Euclide, il y avait là un langage commun et des pratiques, sinon unitaires, du moins transversales aux différentes spécialités : intercaler un ou des moyens, inverser et permuter les termes... Mais la théorie des médiétés, en tant que telle, a semble-t-il été reconnue seulement après les travaux d'Eudoxe et d'Ératosthène. En lisant Théon, on pourrait croire que ce dernier a joué un rôle important dans l'interprétation du passage mathématique de l'Épinomis et, comme on l'a vu chez Proclus, les commentateurs ultérieurs semblent également les avoir associés. Il serait néanmoins imprudent de les suivre : il n'y a pas de raison de croire davantage en la fiabilité historique de leur lecture du Platonicien - qui ne

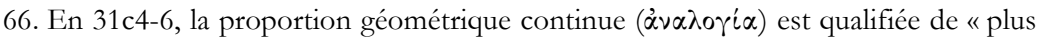

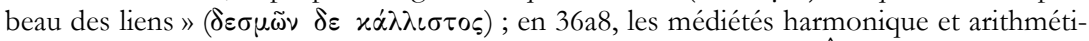
que sont désignées comme des liens des intervalles constitutifs de l'Âme du monde. Il n'y a rien d'étrange à cela : insérer un médian $\mathrm{m}$ entre deux termes $\mathrm{a}, \mathrm{b}$, c'est conjoindre

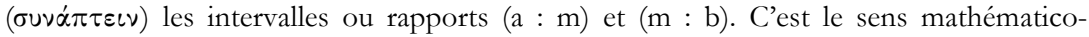
musical, et ce, sans doute bien avant le Timée.

67. Archytas ( $c f$. DK 47 B 2), Théétète (grâce à Pappus, nous savons que les trois médiétés lui servaient à classer les irrationnelles) et Eudoxe (selon Jamblique, In Nicom. arith., 101. 1-6 et Proclus, In Euclidem I, 67.5 Friedlein, peut-être d'après Ératosthène, il aurait découvert les médiétés dites 4 à 6 , ce qui constitue le développement d'une théorie désormais constituée). 
nous est pas parvenu - qu'en leur lecture de l'Épinomis. Du moins, avonsnous ce dialogue et à défaut de savoir ce qu'il y avait dans le premier, nous pouvons voir ce qui n'est pas dans le second! 


\section{BIBLIOGRAPHIE}

Bоoth, N.B. 1957: «Two points of Translation in Plato Epinomis, 990c5-991b4 », Phronesis, 2 (1957), p. 160-161.

BRISson, L. 2005 : «Epinomis : authenticity and authorship », dans : K. Döring, M. Erler, S. Schorn (éd.), Pseudoplatonica: Akten des Kongresses zu den Pseudoplatonica vom 6.9. Juli 2003 in Bamberg, Stuttgart, 2005 (Philosophie der Antike, 22), p. 9-24.

Des PlaCES, É 1935 : «Le passage mathématique de l'Epinomis et la théorie des irrationnelles (990c5-991a4), Revue des études grecques, 48 (1935), p. 540-550. [= Études platoniciennes, 1929-1979, Leyde, 1981 (Études préliminaires aux religions orientales dans l'empire romain, 90), p. 120-130.]

- 1956 : Platon, EEuvres complètes, t. XII, $2^{\mathrm{e}}$ partie : Les Lois, Livres XI-XII, texte établi et traduit par A. Diès ; Epinomis, par E. des Places, Paris, 1956 (Collection des Universités de France).

DÜRING, I. 1932 : Porphyrios Kommentar zur Harmonielehre des Ptolemaios, Göteborg, 1932 (Göteborgs Högskolas årsskr[...], 38, 1932.2).

FESTA, N. 1975 : Iamblichi De communi mathematica scientia liber, ad fidem codicis florentini ed. N. Festa (MDCCCXCI), editionem addendis et corrigendis adjunctis curavit U. Klein, Stuttgart, 1975 (Bibliotheca scriptorum graecorum et romanorum teubneriana, 1443).

HiLLER, E. 1878 : Theonis Smyrnaei philosophici platonici Expositio rerum mathematicarum ad legendum Platonem utilium, recensuit —, Leipzig, 1878 (Bibliotheca scriptorum graecorum et romanorum teubneriana). [Réimpr. Stuttgart-Leipzig, 1995.]

Hoche, R. G. 1866 : Nicomachi Geraseni Pythagorei Introductionis arithmeticae libri II rec. R. Hoche. Accedunt codicis Cizensis problemata arithmetica, Leipzig, 1866 (Bibliotheca scriptorum graecorum et romanorum teubneriana).

Hultsch, F. 1876-1878 : Pappi Alexandrini Collectionis quae supersunt, e libris manu scriptis ed. latina interpretatione et commentariis instr. -, Berlin, 3 vol., 1876-1878 (I. Lib. II-V, rel.; II. Lib. VI-VII, rel.; III. 1: Lib. VIII, rel., schol. suppl.; III. 2 : Indices). [Réimpr. Amsterdam, 1965.]

JONES, R.M. 1932 : "Incommensurable Numbers and the Epinomis», American Journal of Philology, 53 (1932), p. 61-66.

LaCEY, A.R. 1955-1956 : "The Mathematical Passage in the Epinomis», Phronesis, 1 (19551956), p. 81-104.

Leroux, G. 2002 : Platon, La République, traduction inédite, introduction et notes par —, Paris, 2002 (GF, 653).

PISTELLI, E. 1975 : Iamblichi in Nicomachi arithmeticam introductionem liber, ad fidem codicis florentini ed. H. Pistelli (MDCCCXCIV), editionem addendis et corrigendis adjunctis curavit U. Klein, Stuttgart, 1975 (Bibliotheca scriptorum graecorum et romanorum teubneriana, 1444).

Rabouin, D. 2009: Mathesis Universalis : l'idée de "mathématique universelle 》 d'Aristote à Descartes, Paris, 2009 (Épiméthée).

Robin, L. 1950 : Platon, Euvres complètes, traduction nouvelle et notes par - avec la collaboration de M.-J. Moreau, t. II, Paris, 1942 (Bibliothèque de la Pléiade).

TANNERY, P. 1925 : «L'éducation platonicienne. Tableau des sciences mathématiques au temps de Platon» dans: Id., Mémoires scientifiques, publiés par J.L. Heiberg et H.G. Zeuthen, Antiquité, vol. VII, Pbilosophie ancienne (1880-1904), Toulouse-Paris, 1925, p. 1-102 (en partic. «Digression sur un passage de l'Épinomis»). [= «L'éducation platonicienne ", Revue philosophique de la France et de l'étranger, 10 (1880), p. $517-530$; 11 (1881), p. 283-299 ; 12 (1881), p. 151-168, 615-636.] 
Tarán, L. 1975 : Academica : Plato, Philip of Opus and the Pseudo-Platonic Epinomis, Philadelphia, Pa, 1975 (Memoirs of the American Philosophical Society, 107).

TAYLOR, A.E. 1926 : Plato, the Man and his Work, Londres, 1926.

Thomson, W. \& G. Junge 1930 : The Commentary of Pappus on Book X of Euclid's Elements, Arabic text and translation by W. Thomson, with introductory remarks, notes, and a glossary of technical terms by G. Junge and W. Thomson, Cambridge, Mass., 1930 (Harvard Semitic series, 8). [Réimpr. : F. Sezgin (ed.), Islamic Mathematics and Astronomy, vol. 16, Frankfurt am Main, 1997.]

VAN DER WAERDEN, B. L. 1954 : Science awakening, Gröningen, 1954 [traduction anglaise par A. Dresden, "with additions of the author», de: Ontwakende wetenschap: Egyptische, Babylonische en Griekse wiskunde, Gröningen, 1950 (Historische bibliotheek voor de exacte wetenschappen, deel 7].

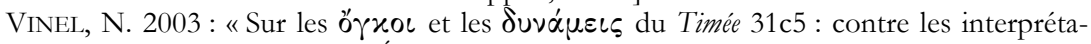
tions modernes », Les Études classiques, 71 (2003), p. 51-70.

VitraC, B. 2005 : «Les classifications des sciences mathématiques en Grèce ancienne », Archives de philosophie, 68 (2005), p. 269-301.

- 2006 : «Les mathématiques dans le Timée de Platon : le point de vue d'un historien des sciences ", Études platoniciennes, 2 (2006), p. 11-78.

— 2008a : «Ératosthène et la théorie des médiétés », dans : Ch. Cusset \& H. Frangoulis (éd.), Ératosthène, un athlète du savoir: journée d'étude du vendredi 2 juin 2006, Université de Saint-Étienne, Saint-Étienne, 2008 (Centre Jean-Palerne. Mémoires, 31), p. 77103.

— 2008b : «Les formules de la "puissance" ( $\delta \dot{v} v \alpha \mu \iota \varsigma, \delta u ́ v \alpha \sigma \vartheta \alpha \iota)$ dans les mathématiques grecques et dans les dialogues de Platon», dans : M. Crubellier, A. Jaulin, D. Lefebvre, P.-M. Morel (éd.), Dunamis: autour de la puissance chez Aristote, Louvain-la-Neuve-Paris-Dudley (Mass.), 2008 (Aristote. Traductions et études), p. 73-148.

Wehrli, F. 1969: Die Schule des Aristoteles: Texte und Kommentar, hrsg. von -, 8, Eudemos von Rhodos, Bâle-Stuttgart, 21969). 